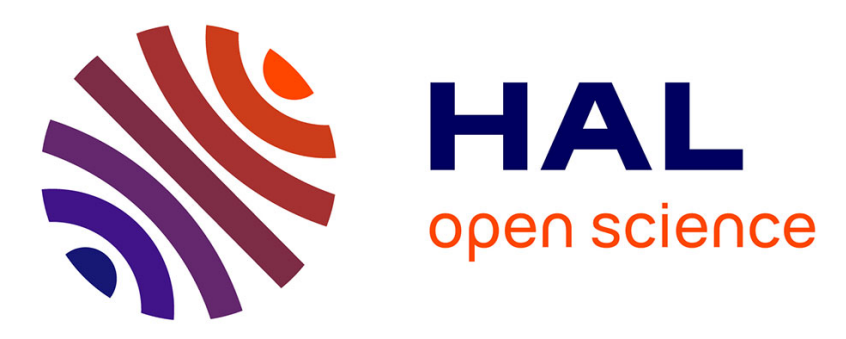

\title{
Logique des frontières, poétique des frontières, antiAtlas des frontières.
}

\author{
Anne-Laure Amilhat Szary, Cedric Parizot, Corinne Fournier Kiss, Patrick \\ Suter
}

\section{- To cite this version:}

Anne-Laure Amilhat Szary, Cedric Parizot, Corinne Fournier Kiss, Patrick Suter. Logique des frontières, poétique des frontières, antiAtlas des frontières.. Patrick Suter; Corinne Fournier Kiss. Poétique des frontières dans les littératures de langue française (XXe-XXIe siècles), MetisPresses, pp.305-350, 2021. halshs-03101137

\section{HAL Id: halshs-03101137 \\ https://shs.hal.science/halshs-03101137}

Submitted on 6 Jan 2021

HAL is a multi-disciplinary open access archive for the deposit and dissemination of scientific research documents, whether they are published or not. The documents may come from teaching and research institutions in France or abroad, or from public or private research centers.
L'archive ouverte pluridisciplinaire HAL, est destinée au dépôt et à la diffusion de documents scientifiques de niveau recherche, publiés ou non, émanant des établissements d'enseignement et de recherche français ou étrangers, des laboratoires publics ou privés. 
Logique des frontières, poétique des frontières, antiAtlas des frontières

Entretien avec Anne-Laure Amilhat Szary et Cédric Parizot

\section{Corinne Fournier Kiss et Patrick Suter}

Anne-Laure Amilhat Szary ${ }^{1}$ et Cédric Parizot ${ }^{2}$, respectivement géographe et anthropologue, ont fondé et animent l'antiAtlas des frontières, collectif transdisciplinaire réunissant des spécialistes de la frontière, des artistes et des praticiens ${ }^{3}$. Leur triple expertise (géographie, anthropologie, art) les désignait tout particulièrement pour aborder la logique et la poétique des frontières d'un point de vue aussi bien spatial qu'anthropologique et artistique. Cet entretien a été réalisé en septembre 2020.

\section{L'antiAtlas des frontières}

CORINNE FOURNIER KISS \& PATRICK SUTER: Pourriez-vous nous présenter l'antiAtlas des frontières, sa genèse, ses spécificités?

CÉDRIC PARIZOT: L'antiAtlas des frontières a été lancé fin septembre 2011 lors d'un colloque art-science à l'Institut d'Études avancées d'Aix-Marseille Université (IMéRA) ${ }^{4}$. Quatre membres du collectif actuel étaient alors présents: Anne-Laure Amilhat Szary (géographe), Jean Cristofol (philosophe et épistémologue de l'art) Nicola Mai et moi-même (anthropologues). À quatre, nous avons lancé un projet intitulé Frontières $d u X X I^{e}$ siècle. L'enjeu était de réunir des artistes, des chercheurs et des experts, pour penser de façon renouvelée les mutations des frontières au XXI" siècle. Le terme "antiAtlas» n'est apparu que lors de l'organisation du colloque-exposition qui devait clôturer ce projet de recherche en octobre 2013 à Aix-en-Provence. L'objectif était d'amener des artistes et des chercheurs à présenter leurs travaux, leurs réflexions et leurs expérimentations communes.

Mais cet événement a en réalité lancé un processus et une démarche que nous avons poursuivis depuis. Un manifeste, Vers un antiAtlas des frontières, a été rédigé pour l'occasion. Nous y précisions que, sans nous ériger contre les cartes, nous refusions l'idée qu'une compilation systématique de cartes, agrémentée de commentaires, puisse produire une connaissance suffisante des frontières ${ }^{5}$. Nous partions effectivement du principe qu'en associant des démarches d'habitude perçues comme non compatibles (celles des artistes, des chercheur et des experts, qui posent tous des questions différentes avec leurs différents objets), nous pouvions renouveler ce champ d'étude.

L'enjeu est double. D'une part, nous appréhendons les écarts, les perturbations ou les repositionnements que génère l'articulation de pratiques très différentes, pour produire des retours critiques sur nos pratiques, nos objets de recherche et de création. D'autre part, nous nous appuyons sur ces expériences pour nous interroger sur les dispositifs qui organisent notre rapport au monde. Nous nous situons dès lors très loin de la phénoménologie, dans la mesure où nous n'envisageons nullement le sujet comme étant nu et seul dans son environnement. Nous réfléchissons ainsi à la manière dont les cartes produisent les frontières - et donc les objets que nous sommes censés décrire. Nous réfléchissons également à la manière dont certains paradigmes visuels comme le réseau organisent et conditionnent de manière spécifique nos questionnements à propos de ces frontières. Comme l'a montré Anna MUNSTER [2013], le réseau conditionne fortement l'expérience des chercheurs et des artistes dans leur analyse et leur rapport à l'espace et aux limites. 
ANNE-LAURE AMILHAT SZARY: De nombreux collègues et amis nous avaient incités à nous rencontrer, Cédric Parizot et moi, et l'occasion s'en est présentée en septembre 2011. Alors que Cédric lançait le programme "Frontières du XXI ${ }^{\mathrm{e}}$ siècle», hébergé par l'IMéRA, qui favorisait les rencontres art-science, j'organisais moi-même un colloque du réseau Border Regions in Transition (BRIT) entre Genève et Grenoble sur le thème de la "frontière mobile» ${ }^{6}$. L'une des sessions portait sur "Arts et frontières». Cédric y a participé, et un réseau s'est constitué entre Aix-Marseille et Grenoble. II s'en est suivi une initiative qui, même si elle bénéficie d'un appui institutionnel de la part de l'IMéRA à Aix-Marseille Université, ne s'est jamais transformée en grand projet scientifique financé par une institution de recherche. L'antiAtlas repose sur des volontés humaines et sur des rencontres, et, au quotidien, il fonctionne comme un bricolage actif, avec beaucoup de liberté. C'est peut-être ce qui contribue à son intérêt et à son originalité.

Je préciserai que nous avons travaillé avec des personnes qui avaient une expérience de la frontière du fait de leur profession (comme l'a dit Cédric), mais aussi de leurs conditions d'existence. Je pense notamment à celles, migrantes ou exilées, avec lesquelles nous avons mené à Grenoble beaucoup d'expérimentations autour de la dimension sensible des franchissements de frontière et des pratiques migratoires. J'ai l'habitude d'associer toutes ces personnes à celles qui font fonctionner l'antiAtlas des frontières.

L'antiAtlas est un dispositif qui navigue entre les normes, comme en témoigne l'exemple du manifeste Vers un antiAtlas des frontières. Au départ, il s'agissait d'un texte collectif, rédigé à la fois en français et en anglais, destiné à être mis en ligne sur notre site au moment du colloque "antiAtlas». Mais, suite à une sollicitation de collègues nord-américains, il a paru (dans une version à peine modifiée) dans le Journal of Borderlands Studies [PARIZOT et alii 2014], une revue qui ne publie en principe que des articles inédits et relus par les pairs. Par la suite, nous avons été constamment amenés à travailler sur les normes régissant la production des artefacts aussi bien scientifiques qu'artistiques destinés à être lus, regardés, touchés ou expérimentés.

\section{CFK \& PS: Quels sont les membres de l'antiAtlas des frontières?}

CP: L'antiAtlas est un collectif fluctuant, dont le projet n'appartient à personne en propre. II s'agit d'un phénomène dynamique et qui nous a très vite dépassés. Certains membres y ont beaucoup travaillé à certains moments, puis d'autres s'y sont impliqués, et ce ne serait pas rendre justice aux uns et aux autres que de se concentrer uniquement sur les membres fondateurs. Il accueille des chercheurs et des artistes, des commissaires d'expositions et des experts. Il nous est ainsi arrivé d'amener des ingénieurs d'entreprises du complexe militaroindustriel, des militaires ou des douaniers, à discuter avec des artistes qui s'étaient efforcés de hacker les systèmes sécuritaires aux frontières de l'Europe.

CFK \& PS: Combien de personnes l'antiAtlas des frontières touche-t-il ou a-t-il touchées?

ALAS: Le noyau le plus fidèle est constitué d'une dizaine de membres, auxquels il faut ajouter un cercle assez proche de plusieurs dizaines de personnes. Mais l'impact de l'antiAtlas a été beaucoup plus grand, puisqu'il faut compter toutes celles et tous ceux qui ont participé aux colloques et aux expositions (que ce soit en y intervenant ou simplement en y assistant), qui ont suivi des ateliers dans leur cursus universitaire, qui ont visité des expositions, et bien sûr les hommes et les femmes en exil avec lesquels nous avons travaillé.

CFK \& PS: Quels sont les projets de l'antiAtlas des frontières? 
CP: Des origines de l'antiAtlas à nos jours, il y a eu des évolutions assez notables. Au départ, étant nous-mêmes chercheurs, nous avons organisé des séminaires de recherche pour engager des discussions avec les artistes que nous invitions. Ensuite, nous avons diversifié les formats en organisant des expositions ou des colloques-expositions.

Mais nous nous sommes surtout lancés dans des expérimentations pratiques avec les artistes. Nicola Mai a fait œuvre de pionnier avec son projet d'ethnofiction intitulé Emborders, pour lequel il a engagé des acteurs professionnels pour jouer les rôles des personnes sur lesquelles il avait enquêté. À travers ce dispositif, il visait deux choses: il s'agissait de réfléchir à la manière dont les frontières humanitaires s'inscrivent dans les corps ainsi qu'aux revendications d'authenticité, d'objectivité et de crédibilité qui sous-tendent à la fois l'action humanitaire et la recherche scientifique.

À la suite de ce travail, nous avons mené d'autres expérimentations. Anne-Laure AMILHATSZARY, Sarah MEKDJIAN et Gladeema NASRUDDIN ont développé avec des migrants un projet de cartographie collaborative et alternative [2013]. De mon côté, je me suis lancé dans la création d'un jeu vidéo avec Douglas Edric Stanley, un artiste enseignant à l'École supérieure d'art d'Aix-en-Provence. Avec lui et Robin Moretti, nous avons réalisé en 2017 un prototype sur la question de la pratique des frontières en Israël-Palestine. Nous avons également monté des pièces à la croisée du cirque et du théâtre, comme Chronique à la frontière avec Vincent Berhault, que nous avons d'ailleurs pu présenter au colloque Poétique des frontières dans les littératures de langue française.

Enfin, en 2016, avec L'antiAtlas Journal', nous nous sommes lancés dans l'exploration de nouveau formats éditoriaux. Cette revue annuelle est dédiée à une approche radicalement transdisciplinaire des frontières contemporaines. Elle prolonge les réflexions et les expérimentations menées par le groupe de recherche dans le cadre des colloques, des conférences et des expositions, mais elle invite aussi des artistes, des chercheurs et des experts à publier des articles sur les frontières ou des expérimentations à la frontière, au croisement de la recherche, de l'art et de l'expertise.

Dans cette revue, nous rendons compte d'expérimentations tout en explorant de nouveaux formats d'écriture de la recherche. Le format spécifique de L'antiAtlas Journal a été créé de façon ad hoc par un artiste et designer, Thierry Fournier (I'actuel directeur artistique de la revue), qui a pensé des articles-paysages sur de grandes nappes, dans lesquelles le lecteur peut se promener (de préférence avec une tablette). L'antiAtlas Journal propose un format où le texte n'est plus nécessairement premier. Car la disposition et l'articulation spécifiques des médias (photos, vidéos, bandes son, etc.) sur ces nappes fait également sens. On peut donc accéder à ces articles, soit par le texte, soit par ces médias, soit à travers les articulations que l'on peut tisser entre eux.

ALAS: Peut-être faut-il rappeler encore que dès les premières expositions (il y en a eu sept en tout, de Marseille à Bogota), nous avons lancé des appels à projets artistiques. Nous avons ainsi contribué - dans une petite mesure, et avec un financement plus que modeste - à produire des dispositifs artistiques, et nous avons reçu des centaines de propositions (surtout visuelles, mais pas uniquement). À une époque où l'idée de border art ou d'»art des frontières» était moins présente qu'aujourd'hui, l'écho qui nous est revenu nous a confortés dans la direction que nous prenions, en signalant toute l'étendue du champ que nous avions repéré.

Par ailleurs, si nous sommes partis de postures relativement classiques en sciences sociales, la confrontation avec des artistes nous a poussés à interroger la dimension créative de nos 
démarches et à rechercher des pratiques autonomes par rapport à la production scientifique standard. Je vois ici deux aspects différents et complémentaires.

Le premier élément concerne le statut de nos productions de chercheurs et de chercheuses ainsi que notre manière de travailler et notre positionnalité. Les dix ans d'antiAtlas ont coïncidé de mon côté avec un approfondissement des méthodologies féministes, qui m'ont aussi permis d'interroger de façon critique la dimension sensible de la création artistique (affect, toucher, etc.). Ainsi, j'ai été amenée à envisager la production scientifique en tant que performance de nature (quasi) artistique, et à considérer l'importance du corps du chercheur ou de la chercheuse dans sa subjectivité, mais aussi dans le fait que ce corps a faim, a soif, a peur, etc. Bref, j'ai été amenée à réfléchir à cet embodiment de la production de la recherche et aux façons dont celui-ci contribue à la constitution de nos objets, de nos méthodes ou de nos choix conceptuels.

Le second élément découle du premier. Le cadre de l'antiAtlas a été extrêmement propice au franchissement de la frontière entre le champ scientifique et le champ artistique, que toute la période moderne avait considérés comme résolument distincts. En travaillant au côté d'artistes ou de façon indépendante, nous sommes devenus nous-mêmes des expérimentateurs, pour produire des formes qui ne se revendiquent plus seulement comme œuvres scientifiques, mais aussi comme productions artistiques. Ainsi, nous mettons quotidiennement à l'épreuve la notion même que nous travaillons, à savoir la frontière - que l'on gagne à considérer non comme un lieu d'affrontement ou de frontalité, mais de complémentarité, d'interface et de mitoyenneté.

Ainsi, nous avons développé des pratiques de recherche-création, ou de performance as research, comme on le dit en anglais, qui s'efforcent de réunir des démarches de création et d'analyse plutôt que de les opposer. Nous nous distinguons de la mode qui, dans le monde scientifique, consiste à utiliser les démarches art-science comme une façon de rendre la science attractive ou de la valoriser. Dans notre cas, les processus de création et d'hybridation entre des champs que l'on s'ingénie à compartimenter - et que nous essayons au contraire de faire vivre ensemble - nourrissent et transforment en profondeur notre façon de faire de la science.

Ce processus est devenu d'autant plus important que nous avions été encouragés au départ de l'antiAtlas par des acteurs pour lesquels la dimension art-science était importante, cette dimension impliquant aussi un rapport à la technologie. C'est pourquoi nous nous sommes souvent présentés comme un triptyque entre art, science et technologie - la dimension technologique des frontières étant très présente dans notre analyse du devenir des frontières politiques.

À Grenoble, je travaille aujourd'hui surtout avec des partenaires de la danse et du théâtre, alors que Cédric mène essentiellement des projets avec des artistes du jeu-vidéo, du cirque et du domaine sonore. L'un et l'autre, nous ne travaillons plus uniquement avec des praticiens des arts visuels, qui étaient auparavant nos interlocuteurs privilégiés quant à la représentation de la frontière. Dans cette perspective, nous avons peut-être contribué à la mise en place d'une pratique post-représentationnelle de l'analyse de la frontière.

\section{CFK \& PS: Mais comment une telle orientation est-elle perçue dans le monde académique?}

ALAS: De façon variée. Notre travail est considéré d'un côté avec appétence, mais, d'un autre côté, nous sommes confrontés à de réels verrous, qui ne concernent d'ailleurs pas seulement la perspective de l'antiAtlas. De fait, toutes les démarches qui nécessitent de s'éloigner des grilles d'évaluations traditionnelles de la fabrication d'un projet de recherche (ce qu'on appelle désormais un research design!) ou des formes de publication classique 
posent problème lors de phases d'évaluation des carrières, de promotion ou de dépôt de projet. Avec quelques collègues, nous avons prévu d'écrire un article basé sur les refus de projets que nous avions pu essuyer au motif que nous proposions des démarches ouvertes au monde de la création! Il y a donc des blocages à certains moments, mais les expertises varient, et il est possible de comparer cette dualité au rapport qu'ont les juges aux graffitis, les uns trouvant que c'est de l'art, les autres qu'il s'agit d'une dégradation de l'espace public. Cette dualité des juges, nous avons pu la constater à l'égard de personnes avec lesquelles nous avons travaillé. L'un des membres de notre collectif, Gladeema Nasruddin, l'auteur de la maquette en glaise réalisée dans le cadre de l'atelier "Cartographies traverses», était demandeur d'asile au moment de ce travail. S'il a fini par obtenir le statut de réfugié, sa demande avait été rejetée lors d'une première audience, sous prétexte qu'il avait travaillé publiquement avec une caméra vidéo alors qu'il aurait dû «rester discret». Le juge qui a statué en deuxième instance s'était renseigné à son sujet sur Google et avait vu qu'il travaillait avec des artistes et des chercheurs, et cet élément semble avoir joué en faveur de la validation.

Notre démarche produit ainsi des réactions assez polarisées. Mais nous ne nous basons pas sur ces retours pour avancer.

CP: Lorsque je me suis engagé dans la démarche de l'antiAtlas en 2011, je ne me rendais pas compte de tout ce que j'allais en retirer du point de vue scientifique - et bien entendu humain. Je ne voyais pas comment j'allais pouvoir tirer parti de cette entreprise pour aborder mes propres questions sur l'économie de la frontière, sur l'espace et les limites en IsraëlPalestine. J'avais l'impression d'ouvrir un autre champ de recherche parallèle. Et puis, très vite, je me suis rendu compte de toutes les retombées de ce travail avec les artistes, lorsqu'ils deviennent des compagnons de recherche, et j'ai compris tout l'intérêt heuristique que peut avoir l'intégration d'une pratique artistique dans la recherche en sciences sociales. J'ai donc commencé à développer des expérimentations pour mieux comprendre mes sujets de recherche. Ainsi, avec Antoine Vion (sociologue), Matthieu Coulon (géomaticien) et Guillaume Stagnaro (artiste et programmeur), nous avons essayé de mettre sur pied un dispositif numérique qui permettait de traiter des données réseau à l'envers, l'idée étant de ne pas donner au départ une image générale du réseau, mais de le déplier au fur et à mesure. Ce projet n'a pas pu être mené à bien faute de financements, mais j'ai continué sur d'autres pistes, notamment avec la création du jeu-vidéo A Crossing Industry. Progressivement, ces expérimentations et ces rencontres $\mathrm{m}^{\prime}$ ont permis de changer ma manière de voir les choses. Je constate ainsi un grand décalage entre les hypothèses de travail que j'avais formulées en 2015, au moment où je me suis lancé dans mon projet d'habilitation à diriger des recherches, et le texte que j'ai achevé cet été. Le cadre épistémologique sur lequel je m’appuie est également très différent.

\section{Poétique des frontières}

CFK \& PS: Vous accordez l'un et l'autre une attention privilégiée aux frontières ainsi qu'à leurs représentations ou à leurs figurations par l'art. Mais vous êtes respectivement géographe et anthropologue, et nous allons désormais tenter de mieux comprendre avec vous le rôle des frontières, leur logique, leur formation. Vos deux profils nous incitent à aborder deux aspects fondamentaux des frontières: leurs dimensions spatiales et sociales. En préparant cet ouvrage, il nous a semblé que c'étaient là les deux lignes de force fondamentales qui entrent en jeu dans les frontières, et qu'elles leur permettent de jouer un rôle de premier plan dans ce que JeanWilliam LAPIERRE [1995] appelle la "poétique des groupements humains». Partagez-vous une telle conception, et pourrait-on dire que votre point de rencontre est justement celui de 
l'intersection des sciences de l'espace et des sciences sociales, qui mène à une interrogation des frontières?

CP: Permettez-moi de répondre en émettant deux réserves. Tout d'abord, la question de la représentation et de la figuration des frontières par l'art ne se trouve pas au cœur de mes propres réflexions, et ceci pour deux raisons. D'une part, j'envisage la pratique artistique non pas dans sa dimension représentative ou figurative, mais dans sa dimension expérimentale (de mon point de vue, les artistes ne sont pas là pour représenter, mais pour nous amener à nous interroger et à repenser les dispositifs à travers lesquels nous représentons le monde). D'autre part, je ne prends pas les artistes pour des objets, mais comme des compagnons de recherche. En d'autres termes, je travaille avec eux pour mieux comprendre les frontières et les dispositifs qu'elles mobilisent dans le monde, et je pense même que les artistes ne sont pas là simplement comme des observateurs ou des chercheurs, mais qu'ils interviennent, tout comme les chercheurs d'ailleurs. La question est donc de savoir comment nous intervenons quand nous travaillons et essayons de comprendre ces frontières, et, par conséquent, de savoir comment nous intervenons dans la poétique des frontières - si du moins le terme "poétique» est retenu dans son sens étymologique, c'est-à-dire comme production.

Ma seconde réserve est d'ordre épistémologique. Je ne décrirais pas mon travail ou notre travail comme se situant à la convergence des sciences de l'espace et du social. En effet, mon approche anthropologique ne s'inscrit pas dans le cadre d'une anthropologie sociale ou culturelle, ni dans celui d'une "sociologie du social» (pour reprendre les termes de Bruno LATOUR [2007]), mais elle correspond à une anthropologie attentive à la fois aux actants humains et non-humains, et donc, pour reprendre les termes d'Eduardo КOHN (2017), je la décrirais comme une "anthropologie au-delà de l'humain». Ce qui m'intéresse, c'est de comprendre comment s'articulent les trajectoires et les actions de ces actants humains et non-humains pour produire les spatialités dans lesquelles nous nous déployons. Autrement dit, je n'envisage pas le social et l'espace comme deux objets différents, mais plutôt comme deux manières distinctes de parler de processus enchevêtrés. L'espace contient et est constitué en partie par des processus sociaux (uniquement en partie, et je me place à cet égard dans une perspective résolument post-humaniste), tout comme le social est toujours spatialisé et matérialisé.

Ces réserves étant exprimées, il demeure que l'antiAtlas s'intéresse évidemment à la poétique des frontières ou à la poétique des groupements humains - au sens étymologique du terme que j'ai rappelé. Encore faut-il préciser que j'aborde pour ma part la poétique des frontières à un niveau infra-symbolique, en-deçà du langage. De mon point de vue, les frontières - qu'elles soient territoriales, sociales, statutaires ou culturelles - ne sont pas produites uniquement au moyen de modes de communication et de représentation symboliques, conventionnels et institutionnalisés. Bien sûr, ces formes symboliques sont fondamentales, comme l'a montré Fredrick BARTH [1995] dans "Les groupes ethniques et leurs frontières», qui a été publié en français dans le volume préfacé par Jean-William Lapierre auquel vous vous référiez. Les frontières ethniques décrites par Barth s'inscrivent dans des formes de communication conventionnelles et institutionnalisées entre des groupes qui parlent les mêmes langues, et elles ont pour rôle de produire de la différence dans la continuité. De même, les formes de communication et de pratiques symboliques sont centrales dans le cadre de la mise en scène et de la défense des frontières territoriales, ou de la mise en place des dispositifs de contrôle. De nombreuses recherches ont d'ailleurs porté sur ces processus de performativité de la frontière, qui vous intéressent probablement aussi dans vos recherches en littérature. 
Mais - et je voudrais y insister - l'expérience et la production des limites intervient aussi à un niveau infra-symbolique, et ma perspective s'accorde avec celle de KOHN [2017] que je citais tout à l'heure, qui se réfère à la sémiologie de Peirce pour étudier les processus indiciels ou iconiques de la représentation. Dans cette perspective, j'envisage la représentation dans ses différents formats. Les indices comme les icônes sont des signes dont les propriétés sont autres que celles des symboles. Alors que les symboles sont des signes qui tirent leur sens des relations conventionnelles qu'ils entretiennent avec d'autres signes, les indices dérivent leur sens de l'association avec un objet, une pratique ou une série d'icônes. Or ces associations ne sont ni conventionnelles ni fixes, et elles se forment ou se dissolvent dans les habitudes du quotidien, dans des formes de communication vernaculaires instables et fluctuantes. Quant aux icônes, ce sont les signes les plus élémentaires, qui entretiennent un rapport de ressemblance avec ce qu'ils désignent, ou qui, pour être plus précis, impliqueraient de ne pas établir de différence entre les signes et les choses. C'est le cas du phasme, qui ressemble à une baguette de bois, et dont la sémiose, comme le dit Kohn, consiste à gommer une telle différence.

Parallèlement aux processus qui visent à les formaliser ou à les institutionnaliser, les expériences des limites et des frontières se produisent aussi à des niveaux infra-symboliques. C'est le cas lorsque la confrontation répétitive à un obstacle (prenons l'exemple d'un checkpoint volant) vient à être partagée au sein d'un groupe. Elle est alors progressivement coordonnée par ses membres à travers des associations entre des icônes et d'autres indices. On sait par exemple que si l'herbe est tassée à tel endroit, c'est que la Jeep est passée et qu'elle ne repassera pas tout de suite. Ou, au contraire, on sait que si l'on a un ralentissement ou un bouchon, c'est que l'on risque de rencontrer un point de contrôle. C'est à travers ce type d'associations qu'ont lieu les constructions des frontières par les populations qui y sont confrontées. Et si une expérience devient "générale», si elle devient une réalité pour un ensemble de personnes, et si elle est coordonnée à travers un quasi-standard - qui peut être le terme de "frontière» ou de "check point», tous deux assez vagues -, cette expérience sera associée à un symbole.

Mais que fait le symbole? Comme dans tout signe, le signifiant mord sur le signifié, bien qu'en partie seulement. Situés dans une forme de relation partielle, l'un et l'autre se transforment. Le symbole ne va pas seulement coordonner, mais il va aussi reconfigurer l'expérience de la frontière, puisqu'il amène avec lui toute sa charge symbolique, la "frontière» ou le "contrôle» renvoyant à d'autres signes, à d'autres symboles, à d'autres sensations, à d'autres émotions.

Inversement, cette expérience vernaculaire ou très locale de la frontière va modifier le sens attribué à ce symbole, et ce qui est intéressant, quand on analyse ces processus poétiques ceux-là même où a lieu la poétique des groupements humains, de l'espace et des limites c'est de pouvoir appréhender combien les systèmes symboliques sont, pour reprendre les mots d'Eduardo Kohn, de "grands touts ouverts». Ceux sur lesquels nous nous appuyons quotidiennement pour évoquer et réprésenter les frontières n'échappent pas à la règle.

CFK \& PS: En fait, vous rejoignez notre horizon de recherche, puisque nous envisageons le spatial et le social comme deux pôles - mais peut-être vaudrait-il mieux parler de "composantes» - en relation et en interaction l'un avec l'autre, et bien sûr également avec des actants humains et non humains. Par ailleurs, notre intérêt pour la poétique des frontières en littérature nous amène justement à être attentifs aux éléments infra-symboliques, ou implicites, qui ne relèvent pas - ou pas immédiatement - du symbolique et de ses conventions, 
et qui nécessitent un déchiffrement - une herméneutique - pour faire apparaître des éléments cachés ou pliés de la frontière.

ALAS: En géographie, nous travaillons à déconstruire la notion de "groupement humain», ou, du moins, nous rappelons constamment la dynamique constante qui préside au fonctionnement de ces groupements, en évitant de les naturaliser ou de les essentialiser. L'un des enjeux de la géographie contemporaine consiste à montrer qu'au-delà des groupements interindividuels - plus ou moins stabilisés selon les époques et les moments - qui ont une dimension classiquement qualifiée de "territoriale», de nombreuses composantes nonterritoriales entrent également en jeu. En particulier, il est acquis que de plus en plus de personnes vivent de manière multisituée et appartiennent de façon concomitante à plusieurs groupements humains. Le groupement national n'est que l'un de ceux qui font sens aujourd'hui dans la constitution des composantes de l'identité des personnes (notamment dans l'accès à un certain nombre de commodités).

CFK \& PS: Peut-être faut-il préciser à ce propos que ce qui est important dans notre perspective à propos de la "poétique des groupements humains», c'est d'une part qu'elle fait écho à la poétique littéraire, d'autre part qu'elle désigne la "poétique en train de se faire», et non pas son résultat. C'est bien une dynamique qui est en cause, et les frontières apparaissent comme des constructions en cours, qui peuvent évoluer ou être abolies. Par ailleurs, bien sûr, ce n'est pas parce qu'on est membre d'un groupe que l'on ne peut pas appartenir également à un ou à plusieurs autres groupes. Ainsi cette "poétique des groupements humains» peut-elle rendre compte de phénomènes complexes.

ALAS: Nous en arrivons à un point important. Et il faut préciser que l'antiAtlas des frontières ne travaille pas sur la frontière, mais sur les processus de frontiérisation, ou, comme on le dit en anglais, de debordering et de rebordering. La frontière n'existe pas en tant qu'élément stable et fixe, mais en tant que processus, qui peut se matérialiser et être normé de manières différentes selon l'expérience des personnes qui doivent la traverser et qui vont la vivre.

J'ajouterai que la frontière, tout en étant une articulation du social et du spatial, l'est aussi du temporel. Je conçois de plus en plus la frontière comme un espace-temps qui matérialise des normes. La notion temporelle intervient à mes yeux au cœur des malentendus - politiques notamment - sur les questions frontalières. Certes, toutes les sociétés fonctionnent avec un rapport à des limites, mais on a l'illusion que la frontière est un invariant. Or un géographe célèbre, Claude Raffestin, a souvent répété que la frontière est un invariant dont la phénoménologie est infinie. Ceci est vrai des limites, dont les frontières constituent un type bien particulier. Les frontières sont situées dans le temps et dans l'espace, tout en revêtant des utilités sociales très distinctes selon les périodes, et il est important aujourd'hui de travailler à comprendre quelles sont les modalités de l'expérience des frontières selon les lieux et les circonstances. Nous sommes dans une tension entre, d'une part, une représentation de la frontière en voie de mondialisation qui permettrait de vendre les mêmes solutions sécuritaires (ou autres) de la Papouasie-Nouvelle-Guinée aux États-Unis ou au Canada, et, d'autre part, une diversité des modes de "faire frontière" rencontrés sur les terrains d'enquête. On y observe des rapports au temps, à l'espace et aux groupes qui contredisent cette représentation standardisée. Car, jusqu'en ce début de $X \mathrm{XI}^{\mathrm{e}}$ siècle, même si le modèle européen de frontières linéaires dites «westphaliennes» s'est exporté, on constate tout de même la survivance de modalités frontalières extrêmement variées qui hybrident en permanence le modèle. 
CFK \& PS: À propos de temporalité, les $X X^{e}$ et $X X I^{e}$ siècles présentent des stations particulièrement importantes dans l'histoire des frontières. On peut penser à la fermeture des frontières en 1914 avec le déclenchement de la Première Guerre mondiale, qui faisait suite à une période de cosmopolitisme intense dont témoigne entre autres l'intense activité des avantgardes de l'époque; à l'érection des frontières entre l'Est et l'Ouest suite à la Deuxième Guerre mondiale; au démantèlement des empires coloniaux; à l'effondrement du mur de Berlin. On a beaucoup parlé alors - même chez certains géographes - de la disparition des frontières, et l'on a vu se développer une nouvelle phase de la mondialisation, avant le retour des nationalismes dans certaines parties du monde (et, dernièrement, la crise de la covid 19, qui a renforcé de manière inattendue quantité de frontières auparavant très fluides, et qui en a même instauré de nouvelles). Notre intuition est que la problématique des frontières ne saurait disparaître, et que leur disparition ou leur ouverture n'est jamais définitive. Claude RAFFESTIN [1986: 19] définissait d'ailleurs lui-même la frontière comme un "commutateur» permettant de l'ouvrir ou de la fermer, de façon hermétique ou non, et de façon différenciée pour les divers individus ou les divers groupes. Comment percevez-vous l'évolution des frontières?

ALAS: Votre question tombe à pic, car j'ai récemment essayé de mieux comprendre le développement du modèle westphalien de la frontière, dont la communauté scientifique s'accordait à dire qu'il devait être déconstruit, mais qu'il était difficile d'aborder avec des dates pertinentes. Vous vous référez aux avant-gardes du début $d u X X^{e}$ siècle, qui se sont effectivement développées dans une période paradoxale où les frontières restaient ouvertes, et où les voyages devenaient beaucoup plus fréquents. Ces derniers ne concernaient cependant que les élites, et l'époque que vous évoquez est aussi celle où l'on a inventé les papiers d'identité au sens où on les comprend aujourd'hui, comme outils d'identification destinés à contrôler les mobilités et l'accès aux territoires (ceux qui ont existé au préalable étaient plutôt des laisser-circuler visant à protéger ceux qui voyagaient). Ayant été créés pour répondre à une situation d'urgence pendant la Première Guerre mondiale dans le but de contrôler les populations dites «nomades» considérées comme dangereuses (car susceptibles de déloyauté à la nation), leur usage va se généraliser ensuite. Dès cette période d'apparente ouverture, on commence donc à différencier certaines circulations, et l'on assiste à une intense fabrication frontalière, qui va déboucher en particulier sur le déploiement des frontières coloniales (cf. la conférence de Berlin de 1884-85). C'est aussi à cette époque que sont posées à La Haye les bases juridiques du système international qui régit actuellement les frontières (conférences de 1899 et de 1907).

Ceci nous montre bien qu'il s'agit, pendant toute cette période de constitution de la frontière - du moins en tant que limite internationale, la «frontière» pouvant désigner bien d'autres choses -, d'un jeu à somme multiple où la somme des parties est supérieure à 1 , malgré l'existence d'un discours sur l'universalité d'un modèle de délimitation linéaire qui repose sur deux équivalences (si bien qu'on devrait avoir un jeu à somme nulle). Tout d'abord, une frontière internationale constitue en principe une ligne entre deux parties ayant un rôle équivalent quant à la négociation de cette ligne, et même s'il y a eu guerre auparavant, un traité de paix s'ensuit entre deux partenaires eux-mêmes de statut similaire en droit international. En principe toujours, toutes les frontières du monde sont par ailleurs équivalentes entre elles. Mais en pratique, dès le moment où cette convention est inventée au XVII siècle - et jusqu'au début du XXe siècle au moins -, certains des acteurs signataires des textes qui formalisent ces principes ne les respectent pas, bien qu'ils soient contraignants au regard du droit international. Au moment même où les États fabriquent les limites internationales - si l'on accepte de se concentrer sur ces frontières spécifiques que sont les 
frontières internationales -, ces mêmes États qui définissent les frontières s'accordent le droit d'aller investir ailleurs (dans des comptoirs, des zones franches, des colonies) et de déroger à ce principe d'équivalence territoriale qu'ils viennent d'instaurer. Par ailleurs, à l'intérieur de leur propres pays, les États souverains vont également établir des limites - par exemple en régulant la circulation des populations non sédentaires -, et ceci, même dans le cadre de systèmes démocratiques où la souveraineté est détenue par l'ensemble du peuple sans distinction de qualité.

On constate donc une ambiguïté très forte, qui repose sur deux ressorts. Tout d'abord, il faut prendre en compte l'attrait qu'exerce cette figure mathématique qu'est la ligne, qui propose une belle symétrie. C'est une forme caractérisée par son universalité et dont la pureté esthétique attire - si bien qu'on en est venu à préférer les frontières droites, alors même que des géographes avaient montré à l'époque que celles qui fonctionnaient le mieux étaient les plus enchevêtrées. D'autre part, il s'agit là d'une fiction politique très pratique pour essayer de maintenir un minimum de stabilité à l'échelle du monde, tout en se donnant la liberté $d^{\prime}$ 'intervenir là où on en a besoin et quand on en a besoin. Les acteurs étatiques puissants jouent avec le système qu'ils ont construit et déclaré intangible: soit en s'appropriant des espaces (colonisation), soit en négociant des cessions temporaires (comptoirs, zones franches, accaparements contractualisés). En définitive, les relations internationales fonctionnement dans un cadre qui déborde de maintes manières le planisphère classique et ses aplats de couleurs stables: de nombreux processus d'extraterritorialité mettent en évidence le fait que les frontières fonctionnent avec le capitalisme. Bien loin de l'en empêcher, elles créent des différences qui le potentialisent. La fin des territoires proclamée au seuil des années 1990 n'était qu'incantatoire.

C'est pourquoi nous croisons le fer avec certains chercheurs, en particulier en sciences politiques, qui peinent à admettre que la frontière est une fiction, et qui posent souvent le débat de façon binaire, en se demandant si tel ou tel phénomène relève ou non d'un processus territorial au sens où il serait enclos dans des frontières ou les dépasserait. De fait, dans le cas des frontières internationales, la dimension territoriale est bien présente et préside au maillage du monde, mais d'autres éléments s'y ajoutent, qui sont "plus que territoriaux». Souvent, la frontière a été définie comme le lieu du malentendu [LA CECLA 2002], et je pense de plus en plus qu'il faut aujourd'hui la définir comme le lieu de la contradiction. C'est un propos que je développe dans l'ouvrage que je viens de terminer, Géopolitique des frontières. Découper la terre, imposer une vision du monde [AMILHAT SZARY 2020].

$\mathrm{CP}$ : Je souscris tout à fait à la proposition d'Anne Laure sur le fait que la frontière est un lieu de malentendus et de contradictions, ne serait-ce que parce que $s^{\prime} y$ articulent de très nombreuses pratiques différentes. Cependant, je pense qu'il est intéressant de garder à l'esprit que ces contradictions et ces malentendus ne sont pas uniquement réductibles à des oppositions ou à des confrontations. Doreen MASSEY, dans son ouvrage, For Space [2005] propose d'envisager l'espace comme une production résultant de l'articulation de multiples trajectoires et de flux d'actions divergents, disjoints, déphasés les uns avec les autres. Si certains sont connectés, d'autres ne le sont pas. Pourtant, tous contribuent à la production de cette même multiplicité toujours en devenir.

Son approche est intéressante à plusieurs égards. Premièrement, elle insiste sur le fait que les trajectoires des uns et des autres ne sont pas systématiquement connectées, et que si elles le sont, ce n'est pas toujours dans le cadre d'interactions dans l'instant, ou dans un rapport de proximité spatiale, mais à travers des formes de relations bien plus complexes dont les personnes ne sont pas toujours conscientes. Dans ce sens, si la frontière est bien un lieu de 
malentendus et de contradictions, c'est aussi un lieu de méconnaissance et d'ignorance du fait que l'on habite dans l'espace de l'autre.

Deuxièmement, l'approche de Massey permet d'envisager la diversité des modes de représentation et de "mise en acte» de la frontière comme autant de réalités distinctes (j'utilise ici l'expression «mise en acte» dans le sens donné par Mol aux mots enactment, enact). Elle se démarque donc d'une approche constructiviste pour s'inscrire dans un réalisme inspiré de la théorie de la relativité. Le réel est alors appréhendé comme multiple. Son approche est de ce point de vue assez similaire à celle de la philosophe néerlandaise Annemarie $\mathrm{Mol}$, qui insiste sur le fait que lorsque l'on appréhende le monde à travers la pratique, la réalité se multiplie. Dans The Body Multiple [2002], en s'appuyant sur une enquête menée dans une clinique traitant l'arthérosclérose, MoL montre comment la même maladie est «mise en acte» différemment par les patients, les chirurgiens ou les biologistes. D'un cas à l'autre, l'arthérosclérose renvoie à des gestes, à des éléments, à des énoncés et à des temporalités très différentes. La maladie est alors envisagée comme un objet multiple, supérieur à un mais inférieur à plusieurs; mais surtout, comme un objet en devenir construit et transformé continuellement par les différentes instances qui la façonnent et qui s'ignorent souvent. Il est ainsi impossible d'en capturer l'ensemble des propriétés, car ces propriétés ne sont pas finies. La question n'est donc plus uniquement de savoir ce qu'est la frontière, mais comment elle est «mise en acte» et donc actualisée, et surtout comment les personnes et les groupes coordonnent la multiplicité des expériences qu'ils en ont.

ALAS: La frontière est à cet égard un lieu exemplaire, et c'est pourquoi elle constitue un lieu de contradiction, terme crucial dans une perception politique et critique de la frontière, à la fois pour déconstruire les modes de pensée dominants et pour ouvrir des possibles dans l'imaginaire politique du monde (en sortant d'un système de pensée «libéral» qui a anéanti pour ainsi dire toute capacité de proposition adverse). Insérée dans un faisceau de réalités très distinctes, la référence à la frontière déclenche des imaginaires très divers. Et c'est en ceci qu'elle permet des rencontres entre des réels, de l'ordre de la connivence ou de la confrontation, et qu'elle peut devenir un lieu de grande violence. Dans ces univers mouvants, souples et ployants, dans cette constitution du multiple de la frontière, il faut être attentif à cette dimension dialectique.

CP: Il faut effectivement être attentif à ces contradictions, à ces confrontations et aux rapports de pouvoir qu'elles génèrent. Mais je pense également que l'on peut aborder les relations à la frontière, entre ces trajectoires et ces flux d'actions, autrement qu'en termes binaires (du type connivence/confrontation, identité/différence, proximité/distance). Les formes de relations que constituent les frontières permettent non seulement d'envisager des liens entre des trajectoires qui se déploient dans des lieux et des moments distincts, mais également de penser des rapports de continuité et d'identité dans la différence. Tel est l'objet du livre que je suis en train de terminer: Israël-Palestine: au seuil de la frontière. Dans ce livre, j'explore comment des connexions qui n'impliquent aucune interaction directe entre Israéliens et Palestiniens, ni nécessairement de conscientisation de la rencontre ou de la nonrencontre, participent activement à la production des limites et des espaces de ces populations.

Ces connexions sont de trois types. Le premier concerne des relations de "correspondance», terme que j'emprunte à Tim INGOLD, qui s'appuie sur la métaphore épistolaire [2017]. Selon Ingold, lorsque nous écrivons des cartes postales à d'autres personnes, nous nous projetons dans un mouvement longitudinal: les réponses arrivent toujours de façon décalée, nous écrivons sans savoir ce que pense l'autre, en l'absence de l'autre. Nous vivons alors une forme 
d'expectation, en essayant de comprendre l'autre, alors que quand l'autre répond, notre trajectoire n'est plus nécessairement la même. Ce qui est donc intéressant, c'est qu'il est possible de penser des relations entre des individus fondées non plus sur des proximités et des interactions immédiates, mais sur des formes d'articulation décalées dans le temps, et qui pourtant nous relient les uns aux autres.

Le deuxième type concerne des relations d'émergence, qui permettent de penser la différence dans la continuité. En effet, dans ces dynamiques d'émergence, l'univers des contraintes à un niveau 1 organisent les conditions de possiblité à un niveau 2. Et chaque fois que les configurations changent au premier niveau, les conditions de possibilité se transforment au second - et inversement. Ainsi, ces niveaux sont connectés bien qu'ils soient totalement différents l'un de l'autre. Ces dynamiques d'émergence permettent de comprendre comment les actions passées d'une personne ou d'un groupe organisent les conditions matérielles qui cadrent les actions d'une autre personne ou d'un autre groupe à un moment ultérieur.

Enfin, le troisième type de connexion que j'analyse entre Israéliens et Palestiniens concerne les rapports «d'involution». DELEUZE et GUATTARI [1980] mobilisent ce terme pour sortir d'une approche de l'évolution et de la parenté en terme filiation, de lien nécessaire ou d'identité entre des parties. Ce qui est intéressant avec le concept d'involution, c'est qu'il aide à penser que deux trajectoires asymétriques qui n'ont en apparence rien à voir l'une avec l'autre peuvent s'articuler et développer ensemble des formes d'interdépendances sans que les sujets en aient conscience. C'est le cas de la guêpe et de l'orchidée, puisque la guêpe devient l'organe de reproduction de l'orchidée, tandis que l'orchidée devient l'objet de l'orgasme de la guêpe. Voici donc des formes de reproduction et de propagation qui n'impliquent pas de conscientisation, dans lesquelles du lien est créé sans intention, et qui est pourtant vital et constitutif de devenirs et de spatialités.

À travers l'étude de ces différentes formes de connexions, j'étudie la façon dont Israéliens et Palestiniens, sans nécessairement s'en rendre compte, réarticulent leurs trajectoires par rapport à celles des autres, en produisant ainsi d'autres spatialités, d'autres seuils, d'autres limites, dans une réorganisation progressive, à des niveaux très infra-symboliques. L'expérience de la limite est ainsi profondément incorporée et intime, et par conséquent extrêmement forte, jusqu'à devenir le socle de l'ensemble des formes de communication symbolique qui sont mobilisées pour en parler.

Par conséquent, s'il est tout à fait important d'insister sur les conflits ou sur les forces d'opposition pour avoir une approche critique des frontières, il ne faut pas oublier les connexions et les articulations qui s'opèrent de façon non instantanée, à distance, et qui se déploient en deçà des représentations, mais qui sont des connexions véritablement constitutives des espaces dans lesquels nous circulons.

CFK \& PS: Vous insistez tous deux sur la "fiction» qui préside à la construction des frontières, et, avant de poursuivre, il nous semble important de rappeler que le mot "fiction" est en fait la traduction latine de la "poétique» grecque: "poétique» vient de poiein, qui signifie "faire», tout comme en latin, fictio vient de facere. Dans The Interpretation of Cultures, Clifford GEERTZ rappelle d'ailleurs que la fiction, en latin, c'est ce qui est façonné [2000: 15]. Et donc, en accordant une grande importance à la poétique, il nous semble que nous sommes assez proches des positions que vous avez développées.

Mais poursuivons dans la réflexion sur les frontières. Les dernières décennies ont été marquées par de nombreux tournants épistémologiques, entre autres avec le développement de ce que l'on a appelé les "études spatiales» (à partir d'Edward Soja, qui indiquait lui-même 
ce qu'il devait à Henri Lefebvre ou à Michel Foucault). Pourriez-vous en rappeler quelques aspects, souligner des changements de paradigmes? Voyez-vous des ruptures épistémologiques par rapport à d'autres conceptions antérieures des frontières? Comme notre ouvrage porte sur la longue durée du XXe siècle à nos jours, verriez-vous des jalons particuliers durant cette période?

ALAS: Même s'il a connu des précurseurs, le tournant spatial remonte à la fin des années 1980 ou au début des années 1990, à un moment où l'on se posait la question de la différenciation des espaces dans le contexte de la mondialisation. II allait permettre de transformer considérablement la conception de la frontière et, paradoxalement, d'en simplifier ou d'en uniformiser la représentation. Jusqu'aux années 1970, la conception de la frontière qui prévalait était celle de la frontière politique, qui délimitait une aire de souveraineté dans un rapport de binarité très clair basé sur un appareillage juridique extrêmement fort lié au champ d'application de l'État. À côté de ce sens, il existait des usages métaphoriques du mot, qui auraient pu désigner beaucoup d'éléments dont nous venons de parler. Aujourd'hui, au contraire, nous travaillons avec ces différentes acceptions en même temps, et le tournant spatial a permis de penser la question des différentiations spatiales autres que celles régies par les États - à des échelles multiples et à des niveaux différents. Par exemple, ce qui est proche dans l'espace n'apparaît pas nécessairement comme ce qui est le plus connu, et l'on peut vivre très proche de références qui sont spatialement lointaines. Ce faisant, la notion de frontière a été considérablement transformée, et la conception classique de la frontière comme ligne a cédé la place à celle du réseau. Le tournant spatial a ainsi correspondu à un moment de déterritorialisation de la pensée de la frontière, précisément en réfléchissant à sa dimension de réseau.

Les années où a été engagé le tournant spatial correspondent cependant également à un moment où, en droit, la frontière a été considérablement complexifiée, dans la mesure où l'on s'est mis à tracer des limites maritimes ou atmosphériques en se demandant à qui appartenait les mers, le ciel, voire le sous-sol, et en posant de fait la question de la tridimensionnalité du territoire, non plus pensé seulement en tant qu'aire, mais aussi en tant que volume. Des superpositions sont ainsi apparues, si bien qu'un État peut être propriétaire du sol tandis qu'un autre peut l'être du sous-sol, un troisième de la première couche de l'atmosphère, un quatrième de la deuxième couche. Le puzzle des frontières devient de plus en plus complexe.

Dans cette perspective, les géographes sont à la fois très heureux du tournant spatial et très précautionneux à son égard. II amène en effet à une prise en compte plus générale de la dimension géographique du social, mais souvent au prix d'une simplification de ce que représente l'espace réduit à son expression aréolaire. Alors que, de notre point de vue, l'espace n'est plus linéaire, il apparaît en même temps comme une combinaison assez complexe entre le réseau, le territoire et le volume. La question des aires culturelles illustre cette contradiction: la démarche du découpage traduit souvent une extériorité altérisante, comme le montre Edward Saïd à propos de la construction de la notion d" "Orient». Le poids des rapports de pouvoir dans l'expression des relations spatialisées à toutes les échelles ne doit pas être oublié, même lorsque les formes de ces rapports se ramifient dans la complexité que Cédric tente de décrypter.

CFK \& PS: Comment prenez-vous en compte la question de la mobilité des et aux frontières?

ALAS: En géographie, la question de la mobilité de la frontière a été théorisée depuis longtemps, même si la conscience de cette propriété est plus grande aujourd'hui. Par exemple, 
à la fin du XIXe siècle, un Friedrich RATZEL [1988 \& 1882-1891] parle de "frontières mobiles", mais dans le sens où il a une vision organiciste de l'Etat. Si une frontière constitue l'enveloppe extérieure d'un groupe humain, elle doit varier avec l'expansion de ce groupe, et donc pouvoir se déplacer. Si bien que pour lui, la frontière devrait être une bande plus qu'une ligne. Son travail sera repris au début du $X X^{e}$ siècle pour justifier l'idéologie expansionniste de l'Allemagne. De la même façon, pour d'autres raisons politiques, le géographe anarchiste Élisée Reclus affirme au même moment que c'est aux groupes humains de définir leurs frontières, si bien qu'elles devraient aussi, par définition être mobiles [FERRETTI 2014]. Cependant jusqu'aux années 2000, le récit dominant reste celui de la frontière fixe, car la grande majorité des théoriciens de l'espace la conçoivent du point de vue des populations sédentaires. Aujourd'hui, la frontière est constamment prise dans un jeu d'oppositions entre le visible et l'invisible ou le mobile et le fixe, et si les discours majoritaires mettent en avant le spectaculaire et le fixe en minimisant du même coup ce qui se passe d'invisible et de mobile, ces dimensions sont constamment présentes et en interaction.

CP: J'ajouterais que si beaucoup de nos collègues ont travaillé sur la technologie et sur la manière dont elle a modifié notre rapport au monde, ils n'ont pas suffisamment porté attention à la manière dont elle a aussi transformé les espaces de notre monde et de notre corps.

Dans notre condition post-numérique, il convient effectivement de s'interroger sur les nouvelles dimensions de nos espaces et sur la manière dont les frontières doivent être intégralement reconceptualisées. C'est ce qu'avait amorcé William GIBSON dans le cadre de son roman Neuromancien [1985]. Pour envisager une réflexion plus récente, on peut se pencher sur ce que réalise l'AADN (Association pour les Arts et Cultures Numériques) qui mène des recherches combinant géographie et art pour explorer les articulations entre espaces numériques et espaces concrets ${ }^{8}$.

De même, les manières dont nos corps se déploient dans le monde, et donc traversent les frontières, ne peuvent plus être envisagés uniquement à travers leur seule enveloppe biologique. Les féministes (je pense notamment à Donna HARAWAY et à la théorie du cyborg qu'elle a développée dans son manifeste de 1985) posaient déjà des questions extrêmement pertinentes à ce propos. Les traces que nous laissons sur le net, les efforts de data mining que développent un ensemble d'acteurs économiques et politiques, complexifient l'appréhension de ce que nous sommes et la manière dont nous agissons. Comme l'explique Louise MerzEAU [2010: 31], il «n'y a plus d'un côté une identité stable (à protéger ou à exhiber) et de l'autre des données qui circulent, mais le façonnage réciproque et continu d'une présence informationnelle».

Ainsi, tant que l'on ne prendra pas en compte les mutations de nos espaces et de nos corps, nous n'arriverons pas à comprendre les transformations des frontières, des systèmes de contrôle aux frontières, ou encore les modalités de leurs contournements.

Pourtant, en dehors du monde de la recherche, de nombreux acteurs ont déjà saisi ces enjeux. Les praticiens des frontières que sont les policiers et les douaniers sont bien plus au fait de ces transformations, et pour cause. De la même manière, les cartels de drogue ont des sistemistas, c'est-à-dire des ingénieurs dont l'objectif n'est pas seulement de contourner les dispositifs de contrôle mais de s'appuyer sur ces transformations des espaces ainsi que des formes de communication et de circulation pour ouvrir et produire de nouvelles routes et de nouvelles manières d'écouler leurs marchandises. Ils explorent les technologies et les spatialités contemporaines, tout comme ils les transforment à travers leurs interventions. Ils sont un peu comme ces artistes qu'évoquait Jean CRISTOFOL [2016] à propos de la photographie. 
Lorsqu'ils se sont saisis de cette nouvelle technologie, l'enjeu était d'en épuiser toutes les possibilités pour comprendre comment ce nouveau dispositif organisait notre rapport au monde. En somme, la recherche en sciences humaines sur les frontières a encore encore beaucoup de chemin à parcourir pour penser et comprendre ce ce que deviennent les frontières dans notre monde contemporain.

\section{Interprétations de la frontière}

CFK \& PS: Pour approfondir la discussion sur les frontières, nous avons souhaité vous soumettre deux citations de philosophes, qui ont fait date et qui sont sans doute datées, mais qui n'en sont pas moins représentatives du fonctionnement de représentations archétypiques de la frontière (perçue comme mur ou comme pont). Du reste, dans Qu'est-ce qu'une frontière aujourd'hui?, vous dites, Anne-Laure AMILHAT SZARY, que "les murs ont vampirisé les imaginaires de la frontière» [Amilhat Szary 2015: 39]. Le mur semble constituer une image particulièrement commune de la frontière, en tout cas pour un non initié, pour un non spécialiste...

ALAS: ... Pour un non spécialiste, vivant sans doute dans un pays riche, et n'ayant pas trop envie d'en bouger. Pour moi ce qui est préoccupant est que des spécialistes partagent aussi cette vision, construisant par exemple des expositions ou concevant des ouvrages sur les frontières... qui ne parlent que de murs, de manière extrêmement réductrice.

CFK \& PS: Soit. Mais comment réagissez-vous aux deux citations suivantes? La première, d'Ortega y Gasset, porte sur le rôle du mur et est empruntée à La Révolte des masses [1929], sans pour autant aborder la question de la révolte. La deuxième apparaît dans la conférence "Bâtir, habiter, penser» de Heidegger (1951) et aborde la frontière comme "pont»:

\footnotetext{
[La place publique] c'est, purement et simplement, la négation du champ. La place, grâce aux murs qui la bornent, est un morceau de champ qui tourne le dos au reste, qui s'en passe et s'y oppose. Ce champ, plus petit et rebelle, qui se sépare du champ infini et se réserve pour lui-même en s'opposant à l'autre, est un champ aboli et, par conséquent, un espace sui generis tout nouveau, dans lequel l'homme se libère de toute communauté avec la plante et l'animal, les laisse dehors et crée une atmosphère à part, purement humaine. C'est l'espace civil. [ORTEGA Y GASSET 2011: 227]

Le pont est une chose, il rassemble le Quadriparti, mais il le rassemble de telle façon qu'il lui donne un emplacement. À partir de cet emplacement se déterminent les places et les chemins par lesquels un espace (Raum) est aménagé [...]. On appelle Raum, Rum une place rendue libre pour un établissement de colons dans un camp. Un espace est quelque chose qui est ménagé, rendu

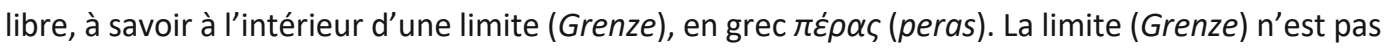
ce où quelque chose cesse, mais bien, comme les Grecs l'avaient observé, ce à partir de quoi quelque chose commence à être. C'est pourquoi le concept est appelé opıçóc (horismos), c'est-àdire limite (Grenze). L'espace est essentiellement ce qui a été ménagé (eingeräumt), ce qu'on a fait entrer dans sa limite. Ce qui a été ménagé est chaque fois [...] rassemblé par un lieu, à savoir par une chose du genre du pont. [HEIDEGGER 1958: 183]
}

ALAS: La citation d'Ortega y Gasset est la plus simple à commenter. Elle pose la question de la matérialisation du lieu de pouvoir, et donc de l'espace public et du rôle qu'y joue la place centrale. Dans la constitution du Nouveau Monde par les puissances hispaniques, le centre des villes est occupé par une place autour de laquelle se concentre le siège des pouvoirs religieux et séculaires, alors qu'en son centre les gens peuvent se rencontrer et prendre des 
décisions, mais "sous le regard de...». II ne me semble pas qu'il soit ici question de l'agora, et Ortega y Gasset évoque d'ailleurs un espace qui tourne le dos au reste du monde, qui établit une frontière entre la civilisation et la barbarie ou l'animalité. Il est certain que les frontières modernes, linéaires, reposent sur ce même principe de binarité caractéristique des Lumières, qui opposent notamment le corps et l'âme. Paradoxalement, ces limites politiques puisent leur force dans leur essentialisation instrumentale, dont l'idée de frontière naturelle est l'illustration parfaite. Aujourd'hui, le recours à la nature pour justifier des limites sécantes est profondément remis en cause, comme l'illustre la question du franchisssement de tous les types de frontières par le virus à l'origine de la pandémie de Covid-19.Vous l'avez souligné, cette citation de La Révolte des masses n'aborde pas la "révolte» contre la frontière. Mais l'idée de révolte, même absente, me permet de rebondir sur le début de notre conversation. La possibilité d'agir contre les frontières implique de nos jours soit de maîtriser la technologie des frontières de façon plus précise que ceux qui les fabriquent ( $c$ 'est le cas de certains trafiquants), soit d'y échapper, par exemple en coupant son téléphone portable, qui est aujourd'hui l'incarnation et la matérialisation de la frontière, puisqu'on y laisse toutes les informations qui nous permettront ou non de franchir des frontières internationales ou autres. Pour constituer «rébellion» - pour reprendre le titre espagnol du livre d'Ortega y Gasset - il y a donc intérêt à se trouver soit dans I'hyper-connexion, soit dans l'hyper-déconnexion (dans les deux cas très loin de la visibilité qu'implique la place publique).

La perspective de Heidegger est très différente, et nous rejoignons pour l'essentiel ses propos selon lesquels la frontière ne se trouve pas là où les choses cessent, mais là où elles se nouent. Plus précisément, les éléments se nouent avec ce que signifie pour chacun d'entre nous la distance. Pendant longtemps, la frontière a été pensée dans une relation linéaire avec la proximité: elle était censée assurer la proximité des membres d'un groupe et les distinguer $d^{\prime}$ 'autres êtres humains qui étaient lointains. Aujourd'hui, la frontière apparaît plutôt comme le lieu de franchissement des distances, comme le montre le virus du SARS-CoV-2, qui traverse aisément les barrières lointaines, et en particulier cette frontière que l'on pensait plus solide qu'elle ne l'est entre l'humain et l'animal, entre le vivant et le non vivant.

CFK \& PS: Cette phrase d'Ortega y Gasset nous paraissait significative du fait qu'il aborde la constitution de l'État, qui se distinguerait du domaine sauvage, en se servant d'une conception propre à l'anthropologie naissante selon laquelle les formes étatiques auraient émergé à partir de "hordes primitives».

Le rapprochement de ces passages d'Ortega et de Heidegger nous a pour sa part retenus dans la mesure où ils exemplifient une opposition structurelle entre des fonctions contradictoires associées à la frontière: fonctions d'ouverture et de jonction du côté du pont, et de fermeture et de disjonction du côté du mur...

CP: Ortega s'inscrit dans un imaginaire inspiré de la physique classique, où le monde est constitué de blocs préexistant les uns aux autres, qu'il est possible de classer, de bouger, de séparer, de découper de manière discrète pour qu'ils n'aient plus aucun lien entre eux. Et c'est là une pensée des limites sans doute datée, mais encore très répandue à l'heure actuelle - je dirais même majoritaire. Sauf qu'il n'en va pas ainsi dans notre monde où l'homme n'est pas coupé de la nature: au contraire, la nature le constitue, tout comme il la constitue. À l'heure de l'Anthropocène, le Grand Partage nature-culture est du reste difficile à défendre.

A propos de la seconde citation, je retiens surtout la conception immersive de l'espace que propose Heidegger, selon laquelle les sujets produisent l'environnement qu'ils perçoivent, l'environnement étant donc lié à la manière dont l'habite le sujet. Bien sûr, Heidegger, en phénoménologue, ne prend pas en compte les dispositifs qui conditionnent la relation entre 
cette personne et son environnement. Mais ce qui m'apparaît intéressant chez lui est son anticipation des conceptions relationnelles de l'espace qui seront développées ultérieurement par Henri Lefebvre, puis par Denis Retaillé ou Doreen Massey. Jon ANDERSON [2012] pousse cette approche jusqu'au bout lorsqu'il envisage la vague (la vague du surfeur) comme lieu, ce lieu étant lui-même compris - dans sa dimension relationnelle, temporaire et éphémère-comme un mouvement. Ainsi le lieu n'apparaît-il pas seulement comme un nœud fixe dans un réseau, mais comme l'articulation dynamique de différentes trajectoires humaines et non humaines qui le créent et qui font sens pour les uns et pour les autres.

ALAS: Nous rejoignons ainsi la question des régimes de frontiérités ou de borderities [AMILHAT SZARY \& GIRAUT 2015]. Plus précisément, il serait erroné d'opposer des frontièresponts à des frontières-murs, car la plupart des frontières sont murs et ponts à la fois. À cet égard, l'espace israélo-palestinien est très particulier, avec des groupes qui ont effectivement des accès très différenciés à la circulation à travers la frontière. L'espace entre les États-Unis et le Mexique est pour sa part stupéfiant, car les habitants y vivent avec un pied de part et d'autre de la frontière (ils peuvent aller à l'école d'un côté et chez le médecin de l'autre, faire les courses tantôt d'un côté, tantôt de l'autre), avec toutefois ce mur qui se dresse au milieu, destiné de fait à un nombre relativement restreint de personnes s'efforçant de le passer de façon illégale dans un sens, mais laissant passer en même temps des armes dans l'autre sens (les États-Unis ayant intérêt à exporter les armes qu'ils fabriquent!). En réalité, la véritable vocation de ce mur est d'être démultiplié dans des images circulant tout autour du monde, dans le but de démontrer qu'une puissance militaire présentée comme "perdue» est ici "retrouvée» dans un contexte civil. Ainsi, ce à quoi ceux qui sont interdits de passage se heurtent, c'est moins au mur lui-même (ils trouvent des façons de le contourner) qu'aux dispositifs d'enfermement et de blocage qu'ils rencontrent ailleurs qu'à la frontière ellemême, une fois qu'ils l'ont passée et qu'ils sont devenus des travailleurs illégaux aux ÉtatsUnis. Par exemple, en allant faire les courses, ils risquent d'être arrêtés pour délit de faciès et d'être déportés en Arizona. Tout se passe comme si le mur s'incarnait dans la couleur de leur peau, qui les distingue par rapport à ce qu'est censé représenter, pour un agent des forces de l'ordre un «Américain national». Le mur ne fonctionne donc qu'avec ce pendant d'un dispositif de contrôle des corps racialisés sur le territoire national [WHITLEY 2015].

Dans cette perspective, ce qui nous lie vraiment entre les membres de l'antiAtlas des frontières, malgré des pensées au fond assez différentes, c'est l'importance que nous accordons aux dispositifs, qui associent des éléments apparemment contradictoires - et notamment ces figures du pont et du mur. Vous noterez cependant que ces termes sont imprécis, et que Heidegger parle d' «une chose du genre du pont». Ainsi, ce qui est important, ce n'est peut-être pas tellement le pont que la question du seuil, du lieu de passage et de I’horizon représenté par ce lieu de passage.

CFK \& PS: Vous avez parlé plus haut de mythe, et c'est en effet parce qu'ils représentent à nos yeux des "grands récits" que nous vous avons présenté ces citations d'Ortega et de Heidegger. Car si LYOTARD a pu parler dans La Condition postmoderne [1979] de la fin des grands récits, il nous semble que ceux-ci continuent très largement de fonctionner.

ALAS: Effectivement, nos frontières européennes et westphaliennes trouvent leur origine dans un schème de pensée qui binarise et qui sépare, et qui a entraîné la distinction entre nature et culture, ou la catégorisation des sciences à l'époque moderne. Dans nombre de cosmologies non occidentales, cette binarité n'existe pas, et on ne dit pas "faire des ponts», pas plus qu'on ne dit «s'affronter», etc. C'est là sans doute quelque chose qui, pour vous, peut 
être source d'interrogations, puisque le volume Poétique des frontières porte sur les écritures francophones, si bien qu'un imaginaire lié au latin est convoqué, avec des notions de front, d'oppositions, etc. Mais cette idée ne se retrouve pas, par exemple, dans l'allemand Grenze, terme qui ne parle ni des ponts, ni de murs mais de bornes...

CFK \& PS: En allemand, on construit bien des ponts ("Brücken bauen» signifie "établir une liaison»), mais il est vrai que les expressions liées à la frontalité sont liées surtout aux langues latines. Le mot allemand "Grenze» est dérivé du mot slave "granica» signifiant la borne. En arabe, le mot frontière حدّ(hadd), toujours utilisé au pluriel حدود (hudud) quand il s'agit de désigner une frontière géographique, renvoie à l'idée de limite et est formé à partir de la racine ح د د (signifiant à la fois aiguiser, rendre coupant, forger et séparer en délimitant). En japonais, de même, le mot frontière signifie limite, l'idéogramme 境 (Kyō) étant composé de la fin (竟) et du sol (土) et pouvant être interprété comme la «fin du sol».

Venons-en à un dernier point: ces deux phrases d'Ortega y Gasset et de Heidegger nous paraissaient exemplaires d'une pensée européenne, et nous nous demandions dans quelle mesure une conception européenne de la frontière pouvait s'opposer à d'autres façons de la considérer dans d'autres régions du monde. Dans notre champ de recherche, nous pensions par exemple à Édouard Glissant, qui parle constamment de "rhizome», de "relation», de "mise en contact». Nous nous interrogions ainsi sur des visions plurielles de la frontière à travers le monde.

ALAS: II y a une question d'anachronisme et d'anaspatialisme qu'il faut essayer d'éviter quand on pense "frontière», car nous sommes prisonniers tant des mots qui expriment les concepts que des façons de les utiliser qui varient selon les époques. Selon Camille LefEBVRE [2015], en tamasheq, en songhay-zarma et en haoussa, le terme utilisé se réfère à l'ouverture d'un corps fermé, donc à quelque chose comme la bouche. Dans d'autres langues subsahariennes, la frontière se dit fin du pouvoir, cette fin n'étant aucunement linéaire. De façon plus générale, il faut rappeler que l'idée ou le concept de frontière peut être universalisante, mais que sa matérialité est toujours très spécifique, si bien que la frontière n'aura pas le même sens suivant que l'on se trouve dans un désert, dans une zone urbanisée, au bord d'un fleuve ou dans des montagnes. À chaque fois, les conditions de passage sont différentes.

Dans les Andes, où j'ai beaucoup travaillé, la règle traditionnelle de l'occupation de l'espace est la circulation entre des environnements distincts pour avoir accès au maximum de ressources, comme c'est le cas dans beaucoup d'environnements montagnards. Et la question principale, c'est la gestion des communs, pas du tout celle de la séparation territoriale. Dans les îles, des limites existent bien sûr, mais les frontières telles que nous les entendons n'ont guère de sens, et il est normal que Glissant ou Chamoiseau le soulignent constamment. Dès lors, on peut se demander ce que signifie tracer une frontière entre Haïti et la République dominicaine: pour le coup, cette frontière est lourde de sens, et elle découle de la colonisation. Dans les archipels, on utilise des cartes, en particulier en coquillages, qui servent à relier les îles, et pas du tout à les séparer.

CP: J'aborderai la question autrement. II me paraît difficile d'associer le concept de frontière à une culture spécifique ou à un lieu particulier, au risque de retomber dans le culturalisme et l'essentialisme.

Certes, les frontières étatiques ont bien été inventées en Europe, mais elles se sont depuis largement propagées, au point de devenir des dispositifs de marquage internationaux quasi universaux. Ce qu'elles sont devenues en Europe n'est plus uniquement européen. D'une part, 
les formes qu'elles prennent aujourd'hui se sont développées grâce aux expérimentations qui ont été menées dans les colonies: le camp, le barbelé sont des expérimentations coloniales. D'autre part, si les Européens ont imposé cette technique de gouvernement durant la période coloniale, les populations colonisées se la sont appropriée. Dans leurs revendications et leurs luttes contre la colonisation, ces populations ont célébré la nation, le territoire et les frontières, ce qui n'empêche pas que les mêmes personnes aient pu faire valoir des pratiques et des représentations différentes de celles des colons. Ceci n'a pas généré uniquement des contradictions, mais aussi des assemblages, des détournements, des reconfigurations qui ont contribué a transformer les sens que les uns et les autres attachaient au concept de frontière.

En outre, il est d'autant plus difficile d'envisager la ou les manières dont on envisage la frontière en Europe que l'on tient compte des mutations que l'on a connues au cours des trente dernières années (en terme de spatialité, de corps, etc.). Comme je le précisais précédemment, l'expérience de l'espace et des corps, et par conséquent celle des frontières, des limites et des formes de circulation, n'a plus rien à voir en 2020 avec celle de 2009, avant l'arrivée du smartphone.

Même si l'on utilise toujours les mêmes mots et les mêmes symboles pour évoquer la question de la frontière, les vécus et les pratiques attachés à ces derniers ont profondément changé. Le rapport d'une personne avec les frontières n'est pas uniquement culturel, mais aussi largement situationnel. Le problème d'une approche culturelle, c'est qu'elle uniformise les manières de vivre et de penser de celles et ceux censés partager la même culture.

Déployer une approche situationnelle ne vise pas à mettre en avant les subjectivités dans toute leur singularité. Je ne pense pas d'ailleurs que notre rapport au monde soit subjectif. Au contraire, comme le disait Haraway, une expérience s'inscrit toujours dans un dispositif de production corporelle, et elle est donc à la fois matérielle et relationnelle. Elle est d'autant plus relationnelle que je ne peux la vivre que si je peux la partager avec d'autres de manière intelligible. Ce qui m'intéresse est donc davantage la spécificité des dispositifs matériels et relationnels qui conditionnent ces expériences de la frontière que le contenu de ces expériences, qui est beaucoup plus difficile à saisir et qu'il faut éviter d'envisager à travers une approche trop déterministe.

Après tout, le terme de "frontière» n'est rien d'autre qu'un quasi-standard, voir un mot d'ordre, c'est-à-dire un terme dont la fonction est de coordonner, voire d'unifier une multiplicité d'expériences et de manières d'habiter le monde. On peut donc parler de vécus au pluriel - des frontières, mais ce ne sont justement que des vécus, qui s'articulent dans des pratiques toujours en devenir, difficilement classifiables, et qui ne permettent pas de constituer des "groupes humains» cohérents et disposant de modes de pensée uniformes et cohérents.

ALAS: C'était l'étape à laquelle j'arrivais. Je ne suis moi-même pas du tout dans une approche culturaliste, mais je pense qu'il est intéressant d'interroger les mots qu'on utilise, et ce qui est important, dans votre question, c'est qu'elle permet de souligner qu'il y a de très nombreuses manières de penser la frontière...

CFK \& PS: Oui, et, pour désigner la frontière, il serait possible de penser par exemple à la membrane, qui est poreuse. Nous en arrivons à évoquer la question des paradigmes qui permettent de la penser.

À ce propos, nous aimerions vous interroger sur les débats contemporains: voyez-vous des lignes de séparation entre des approches opposées des frontières? Naguère, le petit Éloge des frontières de DEBRAY [2010] a beaucoup fait parler de lui, et, plus récemment, les travaux de 
Michel FOUCHER [2016] sur le retour des frontières ont acquis une notoriété certaine. Or vos approches des frontières sont bien différentes. Pourriez-vous vous situer dans ces débats?

CP: Ce qui me distingue de Michel Foucher, c'est mon questionnement anthropologique. Alors qu'il développe une approche très macro-géographique et institutionnelle, j'étudie pour ma part les conditions matérielles à travers lesquelles les populations font l'expérience et produisent ces espaces et ces limites. Concernant Debray, je dirai que je n'entends ni faire l'éloge ni dénoncer les frontières. En tant qu'anthropologue, cela n'aurait pas beaucoup de sens.

ALAS: Le cas de Debray représente une imposture: voici le modèle de l'intellectuel français qui peut parler de tout et en parler bien, en obtenant un large écho à partir d'une simple conférence prononcée au Japon et publiée par un grand éditeur français, simplement parce que le public se demande ce que cet auteur, célèbre pour s'être rendu dans la jungle avec Che Guevara, peut penser des frontières aujourd'hui. Le texte de Debray n'est pas intéressant en lui-même, mais pour ce qu'il signifie de sa propre trajectoire et de celle d'une génération qui avait d'abord mis de côté les frontières, mais qui n'a pas pu ne pas y être confrontée sur le long terme, au point de finalement y revenir pour en faire l'éloge. Du reste, Debray a passablement pillé Foucher, alors qu'il n'avait jamais travaillé sur les frontières d'un point de vue de chercheur.

Foucher a été l'un des premiers à travailler sur la question de la frontière d'un point de vue scientifique, à en faire la géo-histoire, et c'est là un mérite indéniable. En revanche, il ne se départit pas de l'échelle étatique, et ses travaux ne questionnent pas la notion de souveraineté. II a aussi ardemment défendu l'idéal de sa génération, c'est-à-dire le projet européen d'intégration et de partage entre les peuples. Toutefois, il a peu remis en cause son analyse au regard de la sécurisation, de l'individualisation et de la transformation contemporaines des frontières. II s'en tient à une équivalence tautologique entre État, souveraineté, territoire et frontière: le territoire national est celui qui est enclos par les frontières, il est sous l'autorité exclusive de l'État. Son travail est rassurant, parce qu'il contribue à une lecture du monde "tel qu'il est», et bien qu'il travaille sur la construction des frontières et qu'il ait accompli un travail d'inventaire et de géo-histoire nécessaires, tout converge chez lui vers un modèle unifiant qui ne perçoit la frontière que comme ligne. Notre approche différentialiste ou situationnelle est bien différente.

CFK \& PS: Revenons aux questions de terminologie, dont nous avons déjà parlé. Le mot "frontière» est associé à différents autres mots: "front» (dans les langues latines), mais aussi "négociation», "refoulement», etc. Avec quels termes l'associeriez-vous de façon privilégiée?

ALAS: Le mot qui me semble le plus intéressant pour parler de frontière, c'est celui de seuil (que j'ai déjà évoqué), dans la mesure où il signifie la possibilité de communication, mais aussi la différence. La notion même de frontière implique un processus de différenciation qui fait sortir ceux qui y sont confrontés d'un périmètre de sécurité ou de confort. C'est pourquoi le passage d'une frontière n'est pas innocent, et qu'il est fait recours dans certains cas à des rites de passage. Faire frontière pour nous, c'est faire l'expérience $d^{\prime}$ 'un effet de seuil, de distinction, mais aussi de protection par rapport à ce passage.

CP: C'est en effet le seuil qui, à mon avis, permet d'envisager la frontière de la manière la plus stimulante. Le seuil permet d'éviter de réduire la frontière à un objet ou à une étendue dans un espace géométrique. Bien sûr, le «seuil» renvoie à des éléments matériels comme la dalle ou la pièce de bois qui forme la partie inférieure d'une porte, l'entrée d'une maison ou l'élévation d'un terrain. Mais le «seuil» fait aussi référence au point, ou au moment critique 
au cours duquel s'opère une transition, une variation d'intensité, ou encore un changement d'état, et c'est surtout dans cette seconde acception que j'emploie le terme de «seuil» quand je pense aux frontières. Le «seuil» permet donc de penser la frontière dans le mouvement, comme une modulation. Ainsi, lorsque je parle de "seuil», je ne pense pas du tout à une interface.

De ce point de vue, je je me démarque des approches que l'on retrouve dans les postcolonial studies, en particulier chez Homi Bhabha [1995], qui parle de «tiers-espace». Je ne pense pas non plus que la frontière puisse être envisagée comme une étendue disposant d'une certaine épaisseur comme le proposent Aurore MonOd BECQuelin [2012] ou Michel AGIER [2015]. Ces approches tendent à localiser la frontière, à la réduire à certaines de ses manifestations, alors qu'il me semble qu'elle relève davantage de processus délocalisés.

D'ailleurs, même lorsque le terme de seuil renvoie à un objet ou à une dalle (de pierre ou de bois), il est pris dans une structure plus générale. II implique donc une multitude d'autres éléments avec lesquels il entretient des rapports d'interdépendance: ni le seuil ni ces éléments ne tiennent tout seuls. Le seuil constitue les espaces qu'il sépare, tout comme il est constitué par eux. II n'est pas localisé entre eux. II renvoie aux idées de convergence, de voisinage, de coïncidence, de consubstantialité, voire d'indiscernabilité - et c'est à mon sens ce qui est le plus important.

CFK \& PS: Le russe semble donner raison à votre manière de définir la frontière comme seuil, seuil dont vous déployez la signification du côté du mouvement dans le mouvement. En russe, en effet, le mot qui désigne le "seuil», porog (порог), signifie également le "tourbillon» ou le "rapide» sur une rivière...

Les représentations communes des frontières spatiales correspondent pourtant le plus souvent à des lignes, parfois à des points (les fameux "check points»), parfois encore à des zones. Tout à l'heure, Anne-Laure Amilhat Szary a parlé de "réseau». Or c'est également une notion importante dans les études littéraires et pour l'interprétation des frontières, puisque, dans les œuvres, l'herméneutique de la frontière s'appuie souvent sur des éléments qui ne sont pas immédiatement significatifs, mais qui le deviennent dans ces réseaux de signification (des isotopies). Pourriez-vous nous en dire plus? Quelle est l'importance que vous accordez au réseau dans votre interprétation des frontières?

$\mathrm{CP}$ : Tous les termes que vous avez cités renvoient soit à des choses, soit à des éléments de figures géométriques. Or lorsque l'on associe la frontière à des artefacts sécuritaires, à des objets, on a tendance à la réifier, on l'incarne, on la matérialise, on la substantialise. Lorsque l'on évoque des points, des lignes, des réseaux, on a tendance à la schématiser: d'une certaine manière, on dresse une carte en sélectionnant un ensemble de différences significatives, on réalise une abstraction pour essayer de reproduire un autre espace. Dans les deux cas, comme dans beaucoup de réflexions sur l'espace et les limites en sciences sociales, on enferme l'analyse dans une approche visio ou oculocentrée. On reste dans une métaphore visuelle et objectivante, perspectiviste et cartésienne. Ainsi, on n'est pas du tout dans l'approche visuelle de James J. GIBSON [1979], qui est une approche immersive, et qui pense que la représentation n'est pas celle d'un réel qui préexisterait à la représentation, mais une représentation qui constitue le réel. Heidegger n'est pas très loin d'une telle conception.

Pour éviter cette objectivation de la frontière, il est possible de passer par d'autres formes de perception. Lorsqu'on part de l'écoute, le monde n'apparaît plus comme constitué de blocs préexistants ou séparés les uns des autres, mais comme un enchevêtrement de flux. C'est ce que montre Elena BISERNA [2017], qui, en développant une réflexion sur les soundborderscapes, 
souligne combien l'approche par l'écoute rend difficile la conceptualisation de la frontière en termes de rupture, de tiers espace ou d'interface.

Si l'espace est pensé en termes de flux avec lesquels nous sommes en relation, et les limites comme des seuils, les frontières constituent donc des variations dans des continuités. Je les envisage par conséquent comme des multiplicités au sens de DELEUZE et GUATTARI [1980], c'està-dire comme des multiplicités extensives et des multiplicités intensives. Ce sont des multiplicités extensives dans la mesure où elles articulent un ensemble d'éléments et de trajectoires hétérogènes, mais ce sont aussi des multiplicités intensives, dans le sens où elles ne sont pas simplement données à la conscience (elles ne sont pas à l'extérieur) mais sont des éléments de la conscience même. C'est là où le terme de seuil permet d'articuler fondamentalement ces deux dimensions de la multiplicité.

Comme tout modèle visuel, il m'apparaît désormais que la métaphore du réseau (dont nous nous sommes effectivement servi, par exemple dans l'introduction du premier numéro de l'antiAtlas Journal) doit être mobilisée avec précaution, car elle organise notre expérience du monde et notre questionnement de chercheurs de manière spécifique. Pour l'illustrer, je m'appuierai sur la comparaison qu'a proposée Tim Ingold [2026] entre «réseau» et «filet». De manière conventionnelle, le réseau représente des personnes ou des choses, symbolisées par des points, et dont les liens sont schématisés par des fils. Mais cette représentation est statique, car ces liens ou ces interactions interviennent entre des éléments qui conservent toujours les mêmes positions. Or, dans la vie, nos positions ne cessent de changer, alors que nos relations tiennent davantage de correspondances que d'interactions. En outre, ces correspondance sont toujours inscrites dans des dispositifs socio-techniques, même si nous avons l'impression qu'elles sont immédiates. Dans ce sens, le réseau évacue la complexité de la relation et de ce qui la constitue, c'est-à-dire le malentendu, le décalage, ainsi que tout dispositif de médiation. Par ailleurs, les nœuds sont présentés comme des totalités, donnant l'illusion que ces relations concernent l'ensemble des personnes. Or nous ne sommes jamais entièrement en relation avec quelqu'un d'autre, nos "connexions» sont seulement "partielles», comme l'a développé Marilyn StRATHERN [2004]; ce n'est qu'une partie de moi (un moment de ma vie, l'une des mes actions, l'une de mes manière d'être, etc.) qui entre en relation avec quelqu'un, et vice versa.

Le filet permet de présenter d'autres formes de connexions que le réseau, mais il n'est pas parfait non plus. Pour Ingold, les fils du filet représentent les trajectoires des individus ou des éléments en relation, tandis que les relations sont représentées par les mailles. Ce modèle est intéressant à trois égards: 1 . il envisage les personnes comme des trajectoires et non comme des positions fixes; 2 . les relations n'impliquent pas la totalité de la personne mais une partie d'elle-même et un moment précis de sa vie; 3. il permet de penser les relations dans le temps, avec leurs déphasages ou leurs déconnexions et leur partialité.

Le problème du filet, c'est que la trajectoire d'une personne a toujours la même texture, la même couleur, si bien qu'il crée l'illusion de la continuité de l'individu. Or, comme l'a déjà dit Norbert ELIAS en se référant à Hume dans La Société des individus [1991], une telle continuité est elle-même une illusion. Comme les autres modèles visuels, le filet est une carte qui produit une forme d'abstraction [HARRIS \& GAYLE 2019].

Réseau, filet, arborescence, rhizome: tous ces des modèles sont intéressants, mais ce sont des dispositifs qui organisent notre expérience, qui cadrent et formatent notre pensée, en fermant un certain nombre de questions pour en ouvrir un nombre spécifique. Ce qui me paraît intéressant - et c'est ce que nous essayons de faire à l'antiAtlas des frontières -, ce n'est pas de promouvoir un modèle visuel aux dépens d'un autre, mais de jouer sur leurs 
écarts pour découvrir ce qu'ils nous révèlent ou nous permettent de comprendre sur les uns ou sur les autres. Notre démarche est ainsi très artistique, puisqu'il s'agit de jouer constamment avec les formes pour comprendre comment celles-ci organisent notre rapport à la frontière.

\section{Littérature et frontières}

CFK \& PS: Vous vous êtes l'un et l'autre distingués du culturalisme. Mais ne sommes-nous pas enfermés dans notre langue, et ne détermine-t-elle pas en partie nos façons de penser?

ALAS: Oui, et je pense en effet que même si un Tamasheq à Paris ou à Marseille ne perçoit pas la frontière de la même façon, la langue qu'il parle joue un rôle. Du reste, à l'intérieur d'une langue, il peut y avoir de la variété: je pense par exemple à la créolité d'auteurs de la Caraïbe (Glissant ou Chamoiseau). C'est dans ce sens que j'ai apprécié l'approche du colloque Poétique des frontières, qui, en se restreignant au monde de langue française, ouvrait le champ à des perceptions de la frontière venant d'autres lieux que la France. C'est d'autant plus important que la France métropolitaine, parfois avec violence, en empêchant l'usage des autres langues sur son sol, a tout fait pour unifier son langage. Et c'est là que la littérature est intéressante: du fait de son travail sur la langue ou les imaginaires, elle va proposer des éléments qui remettront en question le discours dominant sur les frontières.

CFK \& PS: Ainsi pouvons-nous aborder la littérature, qui constitue l'horizon de ce livre. Et peut-être pouvons-nous préciser un élément. Il est évident que la littérature a une fonction de représentation ou de figuration, mais ceci ne signifie pas qu'elle ne constitue pas en même temps une action. Michel Butor avait l'habitude de dire que sa façon de représenter le monde était une action qu'il exerçait sur le monde, dans la mesure où il travaillait à en modifier les représentations (la modification étant pour lui une préoccupation centrale, comme en témoigne le titre de son roman le plus célèbre). Ainsi a-t-il développé des livres munis de dispositifs typographiques qui entraînent les lecteurs à construire des relations entre des parties du monde éloignées (par exemple entre les hémisphères Sud et Nord dans Boomerang [BUTOR 1978]).

Nous nous demandions si vous étiez justement attentifs à de tels dispositifs littéraires, si vous les utilisiez dans votre travail, dans vos recherches, ou dans votre enseignement.

ALAS: Mon détour esthétique est parti du visuel, qui est un support essentiel du géographe, et la dimension artistique du visuel permet de déconstruire ce qu'il y a de surplombant dans les représentations habituelles, oculocentriques. Cela dit, les arts visuels à propos de la frontière ne sont souvent pas figuratifs, mais conceptuels, et ils passent donc par une dimension textuelle.

Dans les expérimentations de l'antiAtlas, nous avons été de plus en plus attentifs à la dimension performative de nos propres textes, en ayant conscience que nous ne faisions pas que déconstruire les représentations de la frontière, mais que nous contribuions aussi à en produire les représentations. J'ai beaucoup réfléchi pour ma part à ce que les géographes appellent le tournant non représentationnel [THRIFT 2008], qui tente de remettre en cause l'approche discursive de l'analyse des représentations sociales. C'est ainsi que le recours au multimédia s'est déployé, en tant qu'il est le lieu de déplacement du texte vers la performance - et peut-être vers la littérature. J'utilise relativement peu les œuvres littéraires dans mon approche géographique, mais j'aurais plaisir, si nous étions dans la même ville, à donner un cours avec vous, et ce serait certainement le lieu de nombreuses découvertes. En revanche, je retrouve le texte littéraire du côté de l'écriture. II ne s'agit donc pas des textes d'autrui, 
mais de ceux que je peux produire ou que d'autres peuvent produire avec moi. J'utilise le récit, qu'il s'agisse du récit migratoire ou du récit de vie, plus ou moins scénarisé, et il nous arrive d'en susciter dans des ateliers d'écriture, avec différentes méthodes. Et, en ce moment, avec Catherine Boskowitz, une dramaturge qui a beaucoup mis en scène Genet, et dont l'écriture accorde une large place à l'intertextualité, nous travaillons à un texte commun.

Un point néanmoins $m$ 'intéresse beaucoup en littérature: I'intertextualité (peut-être estce dû à mon approche de chercheuse en sciences sociales). À propos des œuvres visuelles, j'ai eu l'occasion de théoriser quelque chose qui est de l'ordre de l'intervisualité. Et, pour ce faire, je me suis beaucoup servie de l'analyse littéraire, de ses méthodes et de ses enjeux, plus que des textes eux-mêmes. Reste que, bien sûr, les représentations littéraires permettent de penser les frontières, et plus encore la fiction, qui n'est cependant pas le propre de la littérature. Dans un module d'un cours que je vais donner cet automne, intitulé "Aimer l'espace», j'utiliserai des évocations littéraires de l'espace, notamment poétiques.

La poésie m'intéresse, en particulier parce qu'elle permet parfois de remettre en cause les catégories de la langue, et les modes de conjugaison. Or la question de la frontière, c'est bien celle de la jonction. Je vous renvoie à un petit livre de David POULLARD et Guillaume RANnou intitulé La Migration. Très précis de conjugaisons ordinaires [2014]. Voici par exemple le verbe «Étranger»: "j'étrange, tu étranges, il étrange», "que j'eusse étrangé»; ou le verbe "À partir de maintenant»: "J'à pars de maintenant, tu à pars de maintenant [...]»; "À pars de maintenant! À partons de maintenant!».

De telles conjugaisons permettent tout de suite de faire comprendre certaines des constructions liées aux frontières. Car la frontière, n'est-ce pas une sorte de lieu de conjugaison? Et de conjugaison problématique?

CFK \& PS: Vous allez donc vers la littérature à partir d'autres expériences, et vous nous rappelez que la littérature elle-même, au XXe siècle et de nos jours, ne se contente souvent pas du texte mais se développe de plus en plus vers la performance ou le multimédia. Le texte littéraire apparaît "hors de lui», franchit les frontières des genres et se réinvente. Ce faisant, la littérature invente d'autres relations entre les domaines de la culture, entre les textes, tout en donnant à penser les frontières et leurs rôles.

Cette question de l'ouverture de la littérature au-delà d'elle-même, dans une transmédialité généralisée, ne fait qu'affleurer dans ce volume. Il y aurait là matière à d'autres études, qui rejoindraient plus nettement encore votre travail au sein de l'antiAtlas des frontières.

Mais, pour terminer, nous aimerions vous interroger sur l'effet boomerang (pour reprendre le titre de Butor) qu'a eu pour vous la participation au colloque Poétique des frontières dans les littératures de langue française. Pourriez-vous nous signaler l'un ou l'autre prolongement qu'il aurait pu susciter dans votre travail?

CP: Ce colloque a confirmé l'intérêt que je nourrissais déjà pour la littérature et sa capacité d'intervention. Car je n'envisage pas la littérature comme une pratique de représentation. A travers les fictions qu'ils composent et les déplacements qu'ils provoquent, les écrivains produisent des expériences, au sens de John DEWEY [2010], qui nous amènent souvent à remettre en jeu notre rapport au monde.

Parfois, ils contribuent même à produire des outils qui s'incarnent dans la réalité. C'est le cas de William GiBSON qui, dans Neuromancien, s'inspire de la pensée cybernétique, ce roman ayant contribué à construire et à diffuser largement les imaginaires que nous mobilisons pour penser l'informatique et les espaces dans lesquels nous vivons dans ce moment postnumérique. 
ALAS: Ce colloque sur la littérature et les frontières m'a tout d'abord interpelée par son titre, puis par la proposition d'une construction commune d'une poétique des frontières. Ayant travaillé dans le cadre d'un projet de recherche internationl avec Johan Schimanski, Stephen Wolfe et Jopi Nyman sur les «border poetics», j'étais très intéressée par la découverte d'une approche francophone de ce domaine. Ma première curiosité allait vers le corpus qui allait faire l'objet d'études, avec une question de ma part un peu naïve sans doute, celle de savoir si la littérature sur la frontière faisait «littérature frontière» ou pas? Je voulais savoir comment on s'attaquait à l'analyse d'un tel corpus, selon quels possibles partages en zones géographiques, au risque de relocaliser les œuvres dans des aires culturelles pourtant mises en question par la perspective même du colloque. De ce point de vue, j'ai été très heureuse de voyager à travers toutes les francophonies au fil du colloque et de noter, autant que je le pouvais, des idées de textes à lire. Car si je ne ne pratique pas l'analyse littéraire, je suis en revanche une grande lectrice (prose, poésie, théâtre), et les textes que je lis inspirent ma pensée, l'irriguent en travaillant mon imaginaire.

Quand au travail des textes, j'ai été très à l'écoute des interventions portant sur les frontières définies comme lignes formelles, mais aussi structurelles ou sémantiques - et sur toutes les manières possibles que les écrivaines et les écrivains ont de passer par-dessus les contraintes, que les limites politiques symbolisent par excellence. Ces manières de «mettre en œuvre» la frontière correspondent en effet aux processus que nous nous évertuons à étudier en tant que chercheuses et chercheurs en sciences sociales. "Il n'est de frontière qu'on outre-passe», écrivait Glissant, et ce qui m'intéresse-là est l'outre: préposition de position, mais aussi sac de peau que l'on emmène avec soi dans le déplacement, pour s'y abreuver, en particulier quand l'eau manque.

Enfin, dans mes recherches sur la production esthétique aux frontières, j'ai été amenée à proposer de relier les œuvres entre elles à travers un paradigme d'intervisualité. J'ai trouvé dans les débats sur la figuration et la transfiguration une grande résonance avec mes propres questionnements. J'ai découvert l'idée de transfictionalité qui m'a également beaucoup inspirée.

De ces lectures explorées suite au colloque, notamment celle de l'œuvre de Michel Butor, je me sers dans mes cours ou dans mon écriture. Mais il y a une autrice en particulier qui m'a beaucoup marquée et dont je suis allée approfondir l'œuvre, il s'agit de Léonora Miano. Aussi, quand l'ami et collègue avec lequel j'ai travaillé à constituer un manuel sur les frontières pour les étudiants, Grégory Hamez, professeur à l'université de Lorraine, m’a parlé de son projet de constitution d'un "Prix Littéraire Frontières» pour lequel il fallait une marraine ou un parrain, je lui ai suggéré Léonora Miano - qui a accepté. Voici donc un résultat concret de votre colloque! L'antiAtlas des frontières a été d'ailleurs sollicité pour faire partie du jury, la boucle est bouclée.

Je voudrais terminer sur cette image du poète Jacques RÉDA [1968:12] à propos de la frontière, que je vous remercie vivement de m'avoir fait découvir, car elle témoigne de cette extraordinaire aptitude de la poésie à capter sans enclore: "La faille de chair du rien qui parle».

\section{Bibliographie}

AMILHAT SZARY, Anne-Laure; MeKDJIAN, Sarah \& NASRUdDIN, Gladeema [2013]. «Un anti-atlas des frontières vécues. Partager la sensibilité des migrants cartographes». URL: https://www.antiatlas.net/colloque-aix-2013-amilhat-szary-mekdjian-gladeema-nasrudeen/ 
AMILHAT SZARY, Anne-Laure [2015]. Qu'est-ce qu'une frontière aujourd'hui? Paris: PUF.

AMILHAT SZARY, Anne-Laure [2020]. Géopolitique des frontières. Découper la terre, imposer une vision du monde. Paris: Le Cavalier Bleu.

Amilhat Szary, Anne-Laure \& GIRAUT, Frédéric (dir.) [2015]. Borderities: the Politics of Contemporary Mobile Borders. Basingstoke: Palgrave Macmillan.

ANDERSON, Jon [2012]. "Relational Places. The Surfed Wave as Assemblage and Convergence», Environment and Planning: Society and Space, vol. 30 (July), no 4, p. 570-587.

antiAtlas Journal, no 1-4 [2016-2020]. URL: http://www.antiatlas-journal.net/

BHABHA, Homi B. [1994]. The Location of Culture. London \& New York: Routledge.

BERTHIER, Aurélien [2015]. «Dans l'épaisseur de la frontière. Entretien avec Michel Agier»,Agir par la culture, no 44, hiver, p.16. URL: https://www.agirparlaculture.be/dans-l-epaisseur-dela-frontiere-entretien-avec-michel-agier/

BARAD, Karen [2007]. Meeting the Universe Halfway: Quantum Physics and the Entanglement of Matter and Meaning. Durham: Duke University Press.

BARTH, Fredrick [1995 (1969)]. «Les groupes ethniques et leurs frontières», in P. POUTIGNAT, \& J. StREIfF-FenART: Théories de l'ethnicité: 203-249. Paris: PUF.

BISERNA, Elena [2017]. "Soundborderscapes: pour une écoute critique de la frontière», antiAtlas Journal \#2. URL: https://www.antiatlas-journal.net/02-soundborderscapes-versune-ecoute-critique-de-la-frontiere.

BUTLER, Judith [2004]. Undoing Gender. New York, London: Routledge.

BUTOR, Michel [1978]. Boomerang. Paris: Gallimard.

Chakrabarty, Dipesh [2000]. Provincializing Europe. Postcolonial Thought and Historical difference. Princeton (N.J.): Princeton University press

CRISTOFOL, Jean; PARIZOT, Cédric \& Amilhat SzARY, Anne-Laure [2016]. «Explorations artssciences à la frontière/Science-Art Explorations at the Borde»r, antiAtlas Journal no 1, 2016. URL: $\quad$ https://www.antiatlas-journal.net/01-introduction-explorations-arts-sciences-a-lafrontiere/.

CRISTOFOL, Jean [2016]. «Arts, Sciences et processus exploratoires», antiAtlas Journal 01. URL: http://www.antiatlas-journal.net/01-arts-sciences-et-processus-exploratoires.

CRISTOFOL, Jean et alii [2017]. Recherche, art et pratiques numériques \#13: de la cartographie alternative à la cybergraphie. URL: https://www.antiatlas.net/tag/cybergraphie/

DEBRAY, Régis [2010]. Éloge des frontières. Paris: Gallimard.

DEWEY, John [2010]. L'art comme expérience. Paris, Folio Essais.

Deleuze, Gilles \& GuATtARI, Félix [1991]. Qu'est-ce que la philosophie? Paris: Minuit.

Deleuze, Gilles \& GuattARI, Félix [1980]. Mille Plateaux. Capitalisme et schiophrénie 2. Paris: Minuit.

Dell'Agnese, Elena \& Amilhat SzAry, Anne-Laure (dir) [2015]. The Aesthetics of Border Demarcation, Geopolitics, no 20-1), p. 1-222.

ELIAS, Norbert [1991], La Société des individus, trad. de I'allemand par Jeanne Etoré, avantpropos de Roger Chartier. Paris: Fayard.

FERRETTI, Federico [2014]. «As Origens da Noção de "Fronteiras Móveis"», Continentes (UFRRJ), vol. 3, no 4, p. 48-65.

FOUCHER, Michel [2016]. Le Retour des frontières. Paris: CNRS Éditions. 
GeERTZ Clifford [2000]. The Interpretation of Cultures. Selected Essays. New York: Basic Books. GiBson, James J. [1979]. The Ecological Approach to Visual Perception.Boston: Houghton Mifflins.

GIBSON, William [1985]. Neuromancien. Paris: La Découverte.

Goody, Jack [1978]. La Raison graphique. La domestication de la pensée sauvage, trad. de I'anglais et présenté par Jean Bazin et Alban Bensa. Paris: Minuit.

HARAWAY, Donna [1985]. "A Cyborg Manifesto. Science, Technology and Socialist-Feminism in the late twentieth Century», Socialist Review, no 80, p. 65-108.

HARRIS, Edmund \& GAYLE, Rhett, "Une ébauche de la théorie des catégories à destination des designers», antiAtlas Journal, no 3, 2019. URL: https://www.antiatlas-journal.net/03-uneebauche-theorie-des-categories-a-destination-designers/.

HEIDEGGER, Martin [1958 (1951)], «Bâtir, habiter, penser», Essais et conférences, trad. de I'allemand par André Préau et préfacé par Jean Beaufret, p. 170-193.

INGOLD, Tim [2016]. Lines. With a new preface by the author. London: Routledge.

INGOLD, Tim [2017]. Correspondences. Aberdeen: University of Abderdeen, 2017 URL: https://knowingfromtheinside.org/files/correspondences.pdf

KOHN, Eduardo [2017 (2013)]. Comment pensent les forêts. Une anthropologie au-delà de I'humain, trad. de l'anglais par Grégory Delaplace. Bruxelles: Zones sensibles.

LA CECLA, Franco [2002]. Le malentendu. Paris: Balland.

LAPIERRE, Jean-William [1995]. «Préface», in Poutignat, P. \& Streiff-Fenart, J. 1995. Théories de l'ethnicité, suivi de Fredrick Barth, «Les Groupes ethniques et leurs frontières», p. 9-14. Paris: PUF.

LATOUR, Bruno [2007]. Changer de société. Refaire de la sociologie, trad. de l'anglais par Nicolas Guilhot et revisé par l'auteur. Paris: La Découverte/Poche.

LATOUR, Bruno [2012]. Enquête sur les modes d'existence. Une anthropologie des modernes. Paris: La Découverte.

LatTe Abdallah, Stéphanie; PARIzot Cédric [2017]. Israël/Palestine. L'illusion de la séparation, (dir.), Aix-en- Provence, Presses universitaires de Provence, coll. Sociétés contemporaines.

LefeBVRE, Camille [2015]. Frontières de sable, frontières de papier. Histoire de territoires et de frontières, du jihad de Sokoto à la colonisation française du Niger, XIXe-XXe siècles. Paris: Presses de la Sorbonne, "Bibliothèque historique des pays d'Islam».

LYOTARD, Jean-François [1979]. La Condition post-moderne. Paris: Minuit.

MASSEY, Doreen [2005]. For Space. London: Sage.

MerzeAU, Louise [2010]. "Habiter I'hypersphère», Documentaliste-Sciences de I'Information, vol. 47, $n^{\circ} 1,2010$, p. 30-31.

Monod BecQuelin, Aurore [2012]. "Introduction», Ateliers d'anthropologie, no 37. URL: http://journals.openedition.org/ateliers/9170.

Munster, ANnA [2013]. An Aesthesia of Networks: Conjunctive Experience in Art and Technology. Cambridge: MIT Press.

MoL, Annemarie [2002]. The Body Multiple. Ontology in medical practice: Durham, NC: Duke University Press.

MORTON, Timothy [2010]. The Ecological Thought. Cambridge, Mass.: Harvard University Press. 
ORTEGA y GASSET, José [1937 (1929)]. La Révolte des masses, trad. de l'espagnol par Louis Parrot. Paris: Delamain et Boutelleau.

PARIZOT, Cédric, Amilhat SZARY, Anne Laure et alii [2013]. "Vers un antiAtlas des frontières», manifeste. URL: https://www.antiatlas.net/vers-un-antiatlas-des-frontieres/

PARIZOT, Cédric et alii [2014]. "The antiAtlas of Borders, A Manifesto», Journal of Borderlands Studies, Taylor \& Francis (Routledge), 29 (4), p.503-512.

Poullard, David \& Rannou, Guillaume [2014]. La migration. Très précis de conjugaisons ordinaires no 5. Paris: Le Monte-en-l'air.

RAFFESTIN, Claude [1986]. «Éléments pour une théorie de la frontière», Diogène, vol. 34, no 134, p. 3-21.

RATZEL, Friedrich [1988 (1897)]. Géographie politique. Genève: Éditions régionales européennes/Economica.

RATZEL, Friedrich [1882-1891]. Anthropogeographie (2 vol). Stuttgart: J. Engelhorn.

RÉDA, Jacques [1968]. «Frontaliers», in Amen. Paris: Gallimard: «Le Chemin», p. 12.

Strathern, Marilyn [2004]. Partial Connexions. Walnut Creek, CA: AltaMira Press.

THRIFT, Nigel [2008]. Non-Representational Theory. Space, politics, affect. London / New York: Routledge.

WHITLEY, Leila Marie [2015]. «More than a Line: Borders as Embodied Sites». Ph.D., London: Goldsmiths, University of London (Centre for Cultural Studies). URL: https://research.gold.ac.uk/12314/1/CUL thesis WhitleyL 2015.pdf.

\section{Notes}

\footnotetext{
${ }^{1}$ Anne-Laure Amilhat Szary est professeure de géographie à I'Université de Grenoble et directrice de Pacte, laboratoire en sciences sociales. Elle a fait partie des membres fondateurs de l'antiAtlas des Frontières et en est aujourd'hui l'une des principales collaboratrices. Son domaine d'expertise porte en particulier sur la frontière $d^{\prime}$ 'un point de vue géographique [AMILHAT SZARY 2015 \& 2020] et sur l'art des frontières, ainsi que sur le développement de la recherche-création.

${ }^{2}$ Cédric Parizot, anthropologue du politique, est chercheur au CNRS et directeur adjoint de I'Institut de Recherche et d'Étude sur le Monde Arabe et Musulman à la Maison Méditerranéenne des Sciences de I'Homme, Aix-Marseille université, à Aix-en-Provence. II a cofondé l'antiAtlas des frontières, dont il assure aujourd'hui la coordination. Spécialiste des frontières et des mobilités en Israël-Palestine [LATTE ABDALLAH \& PARIZOT 2017], il explore les retombées heuristiques de l'intégration de la pratique artistique dans le processus de recherche scientifique.

${ }^{3} \mathrm{Cf}$. le site de l'antiAtlas des frontières (https://www.antiatlas. net/), qui présente entre autres une très intéressante galerie d'œuvres d'art et de vidéos, et qui édite la revue numérique antiAtlas Journal: www.antiatlas-journal.net

4 IMéRA: Institut d'Études avancées d'Aix-Marseille - Exploratoire Méditerranéen de I'Interdisciplinarité. URL: https://imera.univ-amu.fr/

5 Ce manifeste est disponible sur le site de l'antiAtlas des frontières. URL: https://www.antiatlas.net/vers-un-antiatlas-des-frontieres/.
} 
${ }^{6}$ Les contributions en sont réunies sur le site https://sites.google.com/a/iepg.fr/xith-britconference/. Cf. également Amilhat-SZARY \& GIRAUt 2015 et DelL'AgneSE \& Amilhat-SZARY 2015. ${ }^{7}$ URL: http://www.antiatlas-journal.net/

${ }^{8}$ Pour une présentation de ces travaux, cf. CRISTOFOL et alii 2017. 